Review

\title{
Natural Bioactive Compounds from Winery By-Products as Health Promoters: A Review
}

\section{Ana Teixeira ${ }^{1, \dagger}$, Nieves Baenas ${ }^{2, \dagger}$, Raul Dominguez-Perles ${ }^{1}$, Ana Barros ${ }^{\mathbf{1}}$, Eduardo Rosa ${ }^{\mathbf{1}}$, Diego A. Moreno ${ }^{2, *}$ and Cristina Garcia-Viguera ${ }^{2}$}

1 Centre for the Research and Technology of Agro-Environmental and Biological Sciences, (CITAB), University of Trás-os-Montes and Alto Douro (UTAD), Quinta de Prados, 5000-801 Vila Real, Portugal; E-Mails: Anaisabelt90@gmail.com (A.T.); rdperles@utad.pt (R.D.-P.); abarros@utad.pt (A.B.); erosa@utad.pt (E.R.)

2 Phytochemistry Laboratory, Department of Food Science and Technology, National Council for Scientfic Research (CEBAS-CSIC), P.O. Box 164, Campus de Espinardo-Edificio 25, E-30100 Murcia, Spain; E-Mails: nbaenas@cebas.csic.es (N.B.); cgviguera@cebas.csic.es (C.G.-V.)

$\dagger$ These authors contributed equally to this work.

* Author to whom correspondence should be addressed; E-Mail: dmoreno@cebas.csic.es; Tel.: +34-968-396-369.

Received: 30 June 2014; in revised form: 19 August 2014 / Accepted: 25 August 2014 / Published: 4 September 2014

\begin{abstract}
The relevance of food composition for human health has increased consumers' interest in the consumption of fruits and vegetables, as well as foods enriched in bioactive compounds and nutraceuticals. This fact has led to a growing attention of suppliers on reuse of agro-industrial wastes rich in healthy plant ingredients. On this matter, grape has been pointed out as a rich source of bioactive compounds. Currently, up to 210 million tons of grapes (Vitis vinifera L.) are produced annually, being the $15 \%$ of the produced grapes addressed to the wine-making industry. This socio-economic activity generates a large amount of solid waste (up to $30 \%, w / w$ of the material used). Winery wastes include biodegradable solids namely stems, skins, and seeds. Bioactive compounds from winery by-products have disclosed interesting health promoting activities both in vitro and in vivo. This is a comprehensive review on the phytochemicals present in winery by-products, extraction techniques, industrial uses, and biological activities demonstrated by their bioactive compounds concerning potential for human health.
\end{abstract}


Keywords: Vitis vinifera L.; grape pomace; phytochemicals; bioactivity; extraction; food industry; pharmaceutical; phenolic compounds

\section{Introduction}

Grape crops are one of the main extended agro economic activities in the world with more than 60 million tons produced globally every year. Thus, for example, 67 million tons of grapes were produced in 2012, with almost 23 million tons corresponding to European contributors [1]. This production is mainly addressed to fresh consumption as table fruit, juice, and raisins. In addition to these major uses, an important proportion of grape production is addressed to vinification processes, which constitutes a relevant traditional activity in several countries in Southwestern Europe (i.e., France, Greece, Italy, Portugal, and Spain), as wine is one of the most consumed alcoholic beverages in the world [2].

Wine production entails the generation of huge amounts of by-products mainly consisting in organic wastes, wastewater, emission of greenhouse gases, and inorganic residues [3]. After grape juice extraction the remaining pomace and stems are currently not valued as highly profitable waste, being mainly directed to composting or discarded in open areas potentially causing environmental problems [4]. Thus, the increasing demand for environment-friendly industrial production in addition to the challenge for gaining operational efficiency and minimizing by-product treatment cost in the wine industry has started to move this sector towards the adoption of preventative integrated waste approaches [5]. However, when prevention is not feasible, the development of innovative procedures to recycle, reuse, and recover these residues is consistent with the growing demand of green materials and renewable resources of nutrients and bioactive compounds for the feed/food, pharmaceutical, and cosmetic sectors, which will allow a reduced dependence on the current manufacturing activity with the starting raw materials. In this sense, the valorization of these wastes will provide further alternatives to reduce the environmental impact of winery activity.

The potential of the valorization of these agro-industrial by-products is currently supported by the extensive information available on their content of healthy promoting phytochemicals with valuable activity concerning to the prevention of oxidative reactions, cardiovascular problems disease, inflammatory processes, and degenerative pathophysiological state developed in adults [6-8].

The absolute amount as well as the relative proportion of these beneficial compounds in vinification residues is conditioned by a myriad of factors including genetic load of the separate grape varieties, agro-climatic conditions, fertilization procedures, and soil properties, among others. On the other hand, the specific wine-making processes as well as the time between the generation of waste and valorization activities, as well as the characteristics of the recycling and recover procedures have a direct impact on the final concentration of phenolic compounds in the material and, therefore, on the potential as a source of bioactive phytochemicals [9-11].

The secondary metabolites including phenolic acids, flavanols, flavonols, anthocyanins, and stilbenes [12], differ in their solubility and recovery yield, which complicates their individual or targeted extraction. In this sense, innovative and more efficient solvents and extraction methods such 
as high pressure and temperature extraction, supercritical fluids, or ultrasound and microwave assisted extractions have been reported in an attempt to enhance the efficiency of the extraction of phytochemicals from vinification residues [13-16].

The main objective of the present work is to compile a comprehensive update and review on the composition and functional aspects of winery by-products, providing detailed information about the valuable bioactive phytochemicals of these multipurpose materials. The technological advancements applicable for the potential recovery of valuable components, from wine-making industry wastes, have been also considered.

\section{Main By-Products Derived from Winery Industry}

The winemaking process is based on ancestral procedures, being more an art than a science. As a general rule, many artisanal practices are strongly rooted in the traditional vinification processes, as well as limited human resources or physical infrastructures during the production operations, limiting the update of the technological advances addressed to minimize waste production in several wine industries [3]. Therefore, implementation of waste management in the wine industry is a challenging task, making necessary the development of innovative and effective valorization procedures. In this sense, the growing demand of final products and the urgency of avoiding the environmental footprints of this agro-industrial activity have encouraged a tough legal framework to ensure the efficiency of the processes and to support the improvements of the recovery and recycling procedures.

The type of residues produced is closely dependent on the specific vinification procedures, which also affect the physic-chemical properties of the residual material, the characteristics of which determine its further use and specific valorization circuit in which it could be integrated. The major residues from wine-making activity are represented by: organic wastes (grape pomace, containing seeds, pulp and skins, grape stems, and grape leaves), wastewater, emission of greenhouse gases $\left(\mathrm{CO}_{2}\right.$, volatile organic compounds, etc.), and inorganic wastes (diatomaceous earth, bentonite clay, and perlite) [17]. In this regard, it is estimated that in Europe alone, 14.5 million tons of grape by-products are produced annually [18].

The valorization of winemaking by-products is mainly represented by the elaboration of soil fertilizers as well as a fermentation substrate for biomass production and livestock feeds [19-21]. However, there are several constraints for current available options for reusing these unprofitable materials. For example, certain polyphenols present in winery by-products are known to be phytotoxic and display antimicrobial effects during composting, impairing their utilization for this purpose. Regarding their use in livestock feed, some animals show intolerance to certain components, such as condensed tannins, which negatively affect digestibility [22]. Hence, their valorization as a source of bioactive phytochemicals of application in pharmaceutical, cosmetic, and food industries might constitute an efficient, profitable, and environment-friendly alternative for residues [23].

\subsection{Grape Pomace or Press Residues}

Grape pomace is the winery waste originated during the production of must (grape juice) by pressing whole grapes. Currently, 9 million tons of this organic residue are produced per year in the world [10], which constitutes $20 \%(w / w)$, on average, of the total grapes used for wine production [24-27]. The 
reported differences on the relative proportion of grape pomace in comparison with the total amount of grapes used during the vinification process is due to the complete material considered as well as the incorporation or not of grape stems as part of the vinification residues when calculating the relative proportion [24-27].

Concerning the general composition of grape pomace, the moisture percentage varies from $50 \%$ to $72 \%$ depending on the grape variety considered and its ripening state. The insoluble residues from this material have a lignin content ranging from $16.8 \%$ to $24.2 \%$ and a protein content lower than $4 \%$. In general, peptic substances are the main polymer-type constituent of the cell walls present in grape pomaces, ranging from $37 \%$ to $54 \%$ of cell wall posysaccharides. Cellulose is the second type of cell wall polysaccharides in abundance in grape pomaces, varying from $27 \%$ to $37 \%$ [28]. Due to this content in non-digestible polysaccharides, additional fermentation processes may be required to avoid gastrointestinal disturbances when this material is integrated within complex food/feed matrices as a form of valorization.

By its composition, grape pomace stands out out as a suitable material to be employed in different processes, in particular the extraction of grape seed oil and polyphenols (mainly anthocyanins, flavonols, flavanols, phenolic acids, and resveratrol), production of citric acid, methanol, ethanol, and xanthan via fermentation, and the production of energy by methanization. Additional uses addressed to obtain alcoholic drinks by short fermentations and distillation, have been also described for this vinification waste [29-31]. Based on its polyphenolic content, several studies have reported a high antioxidant activity of this by-product, suggesting the winery-derived grape pomace as an interesting source for natural antioxidants with application in pharmacological, cosmetic, and food industries [27,32,33].

When considering the separate fractions of grape pomace (seeds and peels), the relative proportion of seeds ranges from $38 \%$ to $52 \%$ of the dry material [34]. Additional reports have pointed out much lower proportion of seeds, which could be almost $15 \%$ of grape pomace $[35,36]$. On this matter, again technological and material management could be responsible for the final composition of this vinification residue. The information available on the composition of grape seeds $(w / w)$ point out the content of up to $40 \%$ fiber, $16 \%$ essential oil, $11 \%$ protein, $7 \%$ complex phenolic compounds like tannins, and other substances like sugars and minerals [37]. Special attention has been paid to the (poly)phenolic content of grape seeds, ranging from $60 \%$ to $70 \%$ of total extractable compounds. This high concentration is of great interest, taking into consideration that during the pressing of grapes only a minor proportion of these compounds is extracted [38], and this fact has attracted the interest of the pharmaceutical, cosmetic, and food industry as a profitable source of natural antioxidants [39-41], such as MegaNatural ${ }^{\circledR}$-Gold Grape Seed Extract by Polyphenolics, and Citricidal ${ }^{\circledR}$ grapefruit seed extract by Nutribiotic.

Grape skins constitute $65 \%$ of the total material of grape pomace on average. Grape skin has been reported as a rich source of phenolic compounds, even though the final yield is dependent on the specific vinification process and the extraction method used (solvent, temperature, time, and other factors) $[8,42,43]$.

The phytochemical profile of this agro-industrial by-product supports its use as an interesting source of bioactive phytochemicals and ingredients. Nevertheless, the lack of appropriate valorization processes makes it mainly used as compost or discarded in open areas potentially causing environmental 
problems. Research on extraction conditions and new designs are needed for optimization of the release of phenolics from grape skins to maximize the properties of the wine pomace [43].

\subsection{Grape Stems}

Grape clusters or grape stems constitute a residue of the winery industry partially applied as a source of astringent compounds, mainly represented by proanthocyanidins [44]. This material is removed before the vinification steps to avoid an excessive astringency of the wine or a negative effect on the organoleptic characteristics. Therefore, their consideration within the vinification process is not absolutely addressed, although this constitutes an indubitable residue produced by the winery industries.

The quantity of stems varies between $1.4 \%$ and $7.0 \%$ of the raw matter processed [45]. Currently, the commercial value of grape stems is low, being mainly used as animal feed or soil amendments. The scarce information available on grape stems composition indicates anyway that this could be a valuable and interesting source of dietetic fiber and antioxidants [23,46-50]. The average moisture percentage of grape stems has been reported as ranging from $55 \%$ to $80 \%$, with the higher variability attributed to the grape variety. The content of grape stems alcoholic insoluble residues is $71 \%$, on average, of the dry matter and no differences between red and white varieties have been observed [28].

Concerning the phenolic composition of grape stems, their content has been shown in flavan-3-ols, hydroxycinnamic acids, monomeric and oligomeric flavonols, and stilbenes [51-53]. In this regard, phenolics from grape stems have been shown to be approximately $5.8 \%$ on a dry weight basis [10].

The current knowledge on the nutritional and phytochemical composition of this plant material should encourage further research that contributes to a greater understanding of its composition and specific outcomes for its application in developing innovative added-value products.

\subsection{Grape Leaves}

To date, leaves from Vitis vinifera L. constitute the less studied or valorized residue of the grape crops and the winery industry. The scarce information available on the grapevine leaves composition informs on their content in organic acids, phenolic acids, flavonols, tannins, procyanidins, anthocyanins, lipids, enzymes, vitamins, carotenoids, terpenes, and reducing or non-reducing sugars [54-58]. The rich and varied chemical composition of these leaves has led to a considerable interest in this plant material as a promising source of compounds with nutritional properties and biological potential. Thus, grapevine leaves are employed in the production of food ingredients and its juice has been also recommended as an antiseptic for eyewash [59,60].

\subsection{Wine Lees}

Wine lees are the residues formed at the bottom of receptacles containing wine, after fermentation, during storage or after authorized treatments, as well as the residue obtained following the filtration or centrifugation of this product. Lees are mainly composed of microorganisms (mainly yeasts), tartaric acid, inorganic matter, and phenolic compounds [61]. Lees play a major role in wine processing as 
they interact with (poly)phenolic compounds, directly related to the colour and other organoleptic properties, and adsorb them [62]. Moreover, lees liberate enzymes favouring the hydrolysis and transformation of (poly)phenolic substrates in phenolics with high added-value and interest like gallic acid or ellagic acid [63]. The scarce literature on this issue has reported the presence of anthocyanins $\left(6-11.7 \mathrm{mg} \cdot \mathrm{g}^{-1} \cdot \mathrm{dw}\right.$ (dry weight)) and other phenolics $\left(29.8 \mathrm{mg} \cdot \mathrm{g}^{-1} \cdot \mathrm{dw}\right)$ in wine lees [61], and Tao et al. described the concentration of total phenolic compounds in wine lees $\left(50 \mathrm{mg} \cdot \mathrm{GAE} \cdot \mathrm{g}^{-1} \cdot \mathrm{dw}\right)[64]$.

\section{Functional Compounds in By-Products from Organic Wineries}

The well-established relationship between diet and health has prompted extensive research concerning the evaluation of the qualitative and quantitative content of bioactive phytochemicals in plant foods and generated side streams (i.e., harvest remains, non-marketable produce, by-products, etc.). These bioactive compounds are secondary metabolites produced by plants under stress conditions such as wounding, infections, and UV irradiation $[65,66]$, and thus these compounds constitute a physiological tool of plants used to maintain health status. Bioactive phytochemicals present in winery by-products are mainly represented by (poly)phenols, which arise biogenetically from two main primary biosynthetic pathways: the shikimate and acetate pathways. These compounds are structurally comprised of one or more aromatic rings bound to distinct moieties. Thus, their chemical structures include a range from simple molecules, such as phenolic acids, to complex polymeric structures like tannins $[10,67]$. Additionally in terms of their biological attributions, in grapes and winery by-products, these compounds play an important role in sensorial characteristics (color, aroma, flavor, bitterness, or astringency), fostering new interests in health-promotion as a source of ingredients and additives or technological adjuvants [68], and thus are of great relevance to researchers, producers, processors, and consumers [69].

The various groups of phenolic compounds described so far in winery by-products are not uniformly distributed in the plant material, being distributed within subcellular compartments [70,71] as part of the different metabolic pathways involved in the plant cell defense to stress and pathogens [66]. In addition, phenolic compounds obtained from wine-industry residues belong to different classes, including phenolic acids (hydroxybenzoic acids and hydroxycinnamic acids), flavonoids (flavanols or flavan-3-ols, proanthocyanidins, flavones, and flavonols), and stilbenes [72], which have been reported as responsible for multiple biological effects [73].

The data available on total phenolic contents in vinification residues have been reported by analyzing wastes from different varieties grown under distinct agro-climatic conditions. Grape pomace is one of the most extensively analyzed materials as a whole, as well as for its separate components, mainly skins and seeds $[2,4,8,9,17,18,27,28,30-34]$. Content in total phenolics in seed extracts assessed by colorimetric assays showed a wide range of variation between cultivars ranging from 88.11 to $667.98 \mathrm{mg} \cdot \mathrm{GAE} \cdot \mathrm{g}^{-1} \cdot \mathrm{dw}[74,75]$. These variations have been attributed to the concomitant effect of a number of factors including the genetic potential for polyphenol biosynthesis, the maturation stage, and the agro-climatic conditions [76,77]. On the other hand, the analysis of the phenolic composition of grape skins has revealed very similar contents in total phenolics from different grape varieties of up to $112 \mathrm{mg} \cdot \mathrm{GAE}$ (gallic acid equivalent) $\mathrm{g}^{-1} \cdot \mathrm{dw}[8,78]$. Total phenolics contents have been also estimated in grape stems residues by using colorimetric methods that allow 
recording of concentrations ranging from 26.88 to $35.99 \mathrm{mg} \cdot \mathrm{GAE} \cdot \mathrm{g}^{-1} \cdot \mathrm{dw}$, and variations from one cultivar to another [79]. Distinct extraction procedures, grape varieties, and agro-climatic conditions contributed to differences in the quantified total polyphenols, including tannins and polymeric proanthocyanidins, with increased contents in stems and at least partially explaining the differences between the total phenolics reported in literature [22].

\subsection{Phenolic Acids}

Phenolic acids include benzoic and cinnamic acid derivatives, with hydroxycinnamic acids being the most abundant class in wine industry by-products. These compounds frequently appear in a conjugated form, namely as glycosylated derivatives or esters of quinic acid, shikimic acid, and tartaric acid [72]. To date, the relative proportion of the phenolic acids in winery by-products compounds have been calculated by HPLC analyses, existing data on residues from red and white cultivars that evidence significant differences between grape types (Tables 1 and 2) and the distinct fraction of residues, namely stems, skins, seeds, pomace, or leaves). The genotype appears to be the major factor influencing the relative concentrations of the different phenolic compounds [80].

\subsubsection{Hydroxybenzoic Acids}

Hydroxybenzoic acid derivatives identified so far, in this industrial residue, are mainly represented by $p$-hydroxybenzoic acid, protocatechic acid, tannic acid, vanillic acid, gallic acid derivatives, and syringic acid (Tables 1 and 2) [68,72]. In this regard, gallic acid is described as the most abundant hydroxybenzoic acid derivative in grape stems, skins and, seeds, followed by syringic acid in grape stems, and protocatecuic acid in grape seeds and skins $[65,81,82]$. Gallic acid is especially relevant due to its role as a precursor of hydrolysable tannins [83]. Interestingly, the analysis of grape seeds and pomace from red varieties revealed the protocatechuic acid as the most abundant hydroxybenzoic acid (98.65 $\mathrm{mg} \cdot \mathrm{g}^{-1} \cdot \mathrm{dw}$, on average), with higher concentration than the other hydroxybenzoic acid derivatives described (Table 1). The differences concerning the phenolic profile in grape pomace and its individual constituents may be supported by the remaining grape pulp, as part of the grape pomace, which could provide additional phenolic content. Seeds from white varieties showed high contents in both gallic acid and protocatechuic acid with no significant differences between them [84].

The analysis of the individual hydroxybenzoic acids in the wine by-products considered in this review (Tables 1 and 2) provides established differences between various residues concerning the agro-climatic conditions linked to their primary production. Thus, Singleton et al. (1978), described protocatechic acid as the only hydroxybenzoic derivative acid present in grapes skins, presenting the trans-isomers at higher concentrations than the cis-isomers [85].

\subsubsection{Hydroxycinnamic Acids}

Hydroxycinnamic acids are found in all parts of grape fruit, with the highest recorded content being in the external tissues of the ripe fruit (grape skins). The concentration in hydroxycinnamic acids generally decrease during the ripening process, however, the total amount of this phenolic class increases proportionally to the fruit size. 
The main hydroxycinnamic acids found in grapes and wines are caftaric, $p$-coutaric, and fertaric acids. Caftaric and fertaric acids are mainly found in the trans-form, while a negligible fraction of $p$-coutaric acid has been found in the cis-form. This profile in phenolic acids of organic material derived from the vinification process showed a different composition depending on the specific waste considered and the grape type (red or white) (Tables 1 and 2). Grape stems from both red and white varieties showed trans-caftaric acid as the most abundant compound in this class [65,81]. The trans-isomers presented higher concentrations than the cis-ones in all cases. In this regard, Singleton et al. (1978) assumed that the trans-configuration of phenolic acids is the natural configuration, whereas the cis-form is the product of UV-induced isomerization [85].

Concerning to grape skins, the data available on the hydroxycinnamic acids in red and white varieties suggested the existence of critical differences. Whereas skins from white grapes are dominated by the content in cis-coutaric acid and trans-caftaric acid [82], this residue from red grapes, in addition to displaying much lower concentration of this type of phenolics, is mainly represented by chlorogenic acid [32]. In this way, no hydroxycinnamic acids have been described in seeds from white varieties whereas in those from red ones they were represented by chlorogenic acids in a relevant concentration, ranging from 2.90 to $6.80 \mathrm{mg} \cdot \mathrm{g}^{-1} \cdot \mathrm{dw}$, [33]. In addition, the analyses of the grape pressing residues (grape pomace, which involved both skins and seeds, and a limited amount of grape stems and pulp) could provide interesting information on the phenolic composition of this material to support further rational valorization processes, however, to the best of our knowledge no descriptions of this phenolic class has been published on these vinification residues.

Finally, concerning grapevine leaves, which some authors do not consider as a residue even though they constitute an indubitable plant material produced as consequence of the wine agro-industrial activity, again, it is interesting to highlight the contents in trans-caftaric acids in both red and white varieties [59,86], except in the varieties "Moscatel de Grano Menudo", "Cencibel", and "Shiraz", in which the main acid is trans-coutaric [87].

\subsection{Flavonoids}

Flavonoids are low molecular weight compounds structurally composed by 15 carbon atoms [88]. So far, the presence of flavanols or flavan-3-ols, proanthocyanidins, flavones, flavanols, and anthocyanins has been described in industrial residues from grapes [68]. The separate classes of flavonoids differ in the oxidation degree of the central pyran-ring [89]. A significant biosynthesis of flavonols takes place during grape development and ripening. The greatest concentration of flavonols in grapes has been described 3-4 weeks post-veraison [90]. This physiological process, together with the harvest timing strongly contributes to the final qualitative and quantitative content in bioactive flavonoids in vinification residues and, therefore, the rational valorization procedures.

The comparison of the flavonoids composition among the residues of wine making showed that flavan-3-ols are in similar concentrations in skins and in seeds, whereas anthocyanins are mainly found in the skins [91]. 
Table 1. Content in phenolic acids (analytical technique employed) in winery residues from different red varieties of Vitis vinifera L.

\begin{tabular}{|c|c|c|c|c|c|c|}
\hline Compound & Stem & Skin & Seed & Pomace & Leaves & Lees \\
\hline \multicolumn{7}{|l|}{ Hydroxybenzoic acid } \\
\hline Gallic acid & $\begin{array}{l}1.30-2.40\left(\mu \mathrm{g} \cdot 100 \mathrm{~g}^{-1} \cdot \mathrm{dw}\right. \\
\text { HPLC-DAD) }[92] ; \\
0.07-33.00\left(\mathrm{mg} \cdot \mathrm{g}^{-1} \cdot \mathrm{dw}\right. \\
\text { HPLC-DAD) }[65,81,82]\end{array}$ & - & $\begin{array}{l}6.80-9.80\left(\mathrm{mg} \cdot \mathrm{kg}^{-1} \cdot \mathrm{fw}\right. \\
\text { HPLC-FD) [84] }\end{array}$ & $\begin{array}{l}\leq 3.97\left(\mathrm{mg} \cdot \mathrm{g}^{-1} \cdot \mathrm{dw},\right. \\
\text { HPLC-UV) [17]; } \\
0.05-0.19\left(\mathrm{mg} \cdot \mathrm{g}^{-1} \cdot \mathrm{dw},\right. \\
\text { HPLC-DAD) [32] }\end{array}$ & - & - \\
\hline$p$-Hydroxybenzoic acid & - & - & - & $\begin{array}{l}\leq 6.59\left(\mathrm{mg} \cdot \mathrm{g}^{-1} \cdot \mathrm{dw}\right. \\
\text { HPLC-UV) }\end{array}$ & - & - \\
\hline Protocatecuic acid & - & $\begin{array}{l}1.50-2.40\left(\mathrm{mg} \cdot \mathrm{kg}^{-1} \cdot \mathrm{fw}\right. \\
\text { HPLC-FD) [83] }\end{array}$ & $\begin{array}{l}3.30-8.70\left(\mathrm{mg} \cdot \mathrm{kg}^{-1} \cdot \mathrm{fw}\right. \\
\text { HPLC-FD) [84] }\end{array}$ & $\begin{array}{l}\leq 98.65\left(\mathrm{mg} \cdot \mathrm{g}^{-1} \cdot \mathrm{dw}\right. \\
\text { HPLC-UV) [17] }\end{array}$ & - & - \\
\hline Syringic acid & $\leq 32.20\left(\mathrm{mg} \cdot \mathrm{g}^{-1} \cdot \mathrm{dw}\right.$, HPLC-DAD) [81] & - & - & - & - & - \\
\hline Tannic acid & - & - & - & $\begin{array}{l}\leq 3.85\left(\mathrm{mg} \cdot \mathrm{g}^{-1} \cdot \mathrm{dw}\right. \\
\text { HPLC-UV) [17] }\end{array}$ & - & - \\
\hline Vanillic acid & - & - & - & $\begin{array}{l}\leq 0.59\left(\mathrm{mg} \cdot \mathrm{g}^{-1} \cdot \mathrm{dw}\right. \\
\text { HPLC-UV) [17] }\end{array}$ & - & - \\
\hline \multicolumn{7}{|l|}{ Hydroxycinnamic acid } \\
\hline Caffeic acid & $\begin{array}{l}\leq 0.60\left(\mathrm{mg} \cdot \mathrm{g}^{-1} \cdot \mathrm{dw}, \text { HPLC-DAD) [81]; }\right. \\
0.60-1.90\left(\mu \mathrm{g} \cdot \mathrm{g}^{-1} \cdot \mathrm{dw} ; \text { HPLC-DAD) [92] }\right.\end{array}$ & - & - & - & - & $\begin{array}{l}663\left(\mu \mathrm{g} \cdot \mathrm{g}^{-1} \cdot \mathrm{dw}\right. \\
\text { HPLC-DAD) [61] }\end{array}$ \\
\hline Caftaric acid & - & - & - & - & $\begin{array}{l}\leq 7.32\left(\mathrm{mg} \cdot \mathrm{g}^{-1} \cdot \mathrm{fw}\right. \\
\text { HPLC-UV) [86] }\end{array}$ & \\
\hline cis-Caftaric acid & - & $\begin{array}{l}0.20-0.50\left(\mathrm{mg} \cdot \mathrm{kg}^{-1} \cdot \mathrm{fw}\right. \\
\text { HPLC-FD) }[83]\end{array}$ & - & - & - & \\
\hline trans-Caftaric acid & $\begin{array}{l}0.04-16.10\left(\mathrm{mg} \cdot \mathrm{g}^{-1} \cdot \mathrm{dw}\right. \\
\text { HPLC-DAD) [65,81]; } \\
\leq 40.00\left(\mathrm{mg} \cdot \mathrm{kg}^{-1} \cdot \mathrm{fw} ; \mathrm{HPLC}-\mathrm{DAD}\right)[44]\end{array}$ & $\begin{array}{l}4.90-9.50\left(\mathrm{mg} \cdot \mathrm{kg}^{-1} \cdot \mathrm{fw}\right. \\
\mathrm{HPLC}-\mathrm{FD})[83]\end{array}$ & - & - & $\begin{array}{l}1.40-3.28\left(\mathrm{mg} \cdot \mathrm{g}^{-1} \cdot \mathrm{dw}\right. \\
\text { HPLC-DAD) }[59]\end{array}$ & \\
\hline Chlorogenic acid & - & $\begin{array}{l}\leq 0.23\left(\mathrm{mg} \cdot \mathrm{g}^{-1} \cdot \mathrm{dw}\right. \\
\text { HPLC-DAD) [32] }\end{array}$ & $\begin{array}{l}2.90-6.80\left(\mathrm{mg} \cdot \mathrm{g}^{-1} \cdot \mathrm{dw}\right. \\
\text { HPLC-DAD) [33] }\end{array}$ & - & - & \\
\hline$p$-Coumaric acid & $\begin{array}{l}0.04-0.90\left(\mathrm{mg} \cdot \mathrm{g}^{-1} \cdot \mathrm{dw}, \text { HPLC-DAD) [81]; }\right. \\
0.90-2.20\left(\mu \mathrm{g} \cdot \mathrm{g}^{-1} \cdot \mathrm{dw}, \text { HPLC-DAD) [92] }\right.\end{array}$ & - & - & - & - & $\begin{array}{l}2449\left(\mu \mathrm{g} \cdot \mathrm{g}^{-1} \cdot \mathrm{dw}\right. \\
\text { HPLC-DAD) [61] }\end{array}$ \\
\hline trans-Coumaroyltartaric acid & - & - & - & - & $\begin{array}{l}0.49-1.49\left(\mathrm{mg} \cdot \mathrm{g}^{-1} \cdot \mathrm{dw}\right. \\
\text { HPLC-DAD) [59] }\end{array}$ & \\
\hline Coutaric acid & $\leq 4.50\left(\mathrm{mg} \cdot \mathrm{kg}^{-1} \cdot \mathrm{fw} ; \mathrm{HPLC}-\mathrm{DAD}\right)$ [44] & - & - & - & - & \\
\hline cis-Coutaric acid & - & $\begin{array}{l}0.90-2.70\left(\mathrm{mg} \cdot \mathrm{kg}^{-1} \cdot \mathrm{fw}\right. \\
\text { HPLC-FD) }[83]\end{array}$ & - & - & - & \\
\hline trans-Coutaric acid & - & $\begin{array}{l}3.20-10.00\left(\mathrm{mg} \cdot \mathrm{kg}^{-1} \cdot \mathrm{fw},\right. \\
\text { HPLC-FD) [83] }\end{array}$ & - & - & - & \\
\hline Ferulic acid & $\leq 2.50\left(\mathrm{mg} \cdot \mathrm{g}^{-1} \cdot \mathrm{dw}, \mathrm{HPLC}-\mathrm{DAD}\right)[80]$ & - & - & - & - & \\
\hline
\end{tabular}

HPLC-DAD: High Performance Liquid Chromatography-Diode Array Detector; HPLC-FD: High Performance Liquid Chromatography-Fluorescence Detector; HPLC-UV: High Performance Liquid Chromatography-Ultraviolet Detector; dw, dry weight; fw, fresh weight. 
Table 2. Content in phenolic acids (analytical technique employed) in winery residues from different white varieties of Vitis vinifera L.

\begin{tabular}{|c|c|c|c|c|c|}
\hline Compound & Stem & Skin & Seed & Pomace & Leaves \\
\hline \multicolumn{6}{|l|}{ Hydroxybenzoic acid } \\
\hline Gallic acid & $\begin{array}{l}0.01-0.03\left(\mathrm{mg} \cdot \mathrm{g}^{-1} \cdot \mathrm{dw}, \text { HPLC-DAD) [92]; }\right. \\
1.05-22.60\left(\mu \mathrm{g} \cdot \mathrm{g}^{-1} \cdot \mathrm{dw} ; \text { HPLC-DAD) [65,81] }\right.\end{array}$ & $\leq 1.20\left(\mathrm{mg} \cdot \mathrm{g}^{-1} \cdot \mathrm{dw}, \mathrm{HPLC}-\mathrm{UV}\right)[81]$ & $\begin{array}{l}\leq 1.90\left(\mathrm{mg} \cdot \mathrm{g}^{-1} \cdot \mathrm{dw}, \mathrm{HPLC}-\mathrm{UV}\right)[82] \\
15.70-19.70\left(\mathrm{mg} \cdot \mathrm{g}^{-1} \cdot \mathrm{dw}, \mathrm{HPLC}-\mathrm{UV}\right)[93]\end{array}$ & $<1.98\left(\mathrm{mg} \cdot \mathrm{g}^{-1} \cdot \mathrm{dw}\right.$, HPLC-DAD) [94] & - \\
\hline Gallic acid hexose & - & - & $\leq 0.80\left(\mathrm{mg} \cdot \mathrm{g}^{-1} \cdot \mathrm{dw}, \mathrm{HPLC}-\mathrm{UV}\right)[82]$ & - & - \\
\hline Gallic acid dihexose & - & $\leq 1.10\left(\mathrm{mg} \cdot \mathrm{g}^{-1} \cdot \mathrm{dw}, \mathrm{HPLC}-\mathrm{UV}\right)[81]$ & $\leq 1.20\left(\mathrm{mg} \cdot \mathrm{g}^{-1} \cdot \mathrm{dw}, \mathrm{HPLC}-\mathrm{UV}\right)[82]$ & - & - \\
\hline Protocatecuic acid & - & - & $\begin{array}{l}\leq 6.00\left(\mathrm{mg} \cdot \mathrm{kg}^{-1} \cdot \mathrm{fw}, \text { HPLC-FD) [84]; }\right. \\
<1.10\left(\mathrm{mg} \cdot \mathrm{g}^{-1} \cdot \mathrm{dw} \text {. HPLC-UV) [49] }\right.\end{array}$ & & - \\
\hline Syringic acid & $\leq 0.10\left(\mu \mathrm{g} \cdot \mathrm{g}^{-1} \cdot \mathrm{dw}\right.$, HPLC-DAD $)[81]$ & - & - & - & - \\
\hline \multicolumn{6}{|l|}{ Hydroxycinnamic acid } \\
\hline Caffeic acid & $\begin{array}{l}\leq 0.05\left(\mu \mathrm{g} \cdot \mathrm{g}^{-1} \cdot \mathrm{dw}, \text { HPLC-DAD) [81]; }\right. \\
1.00-1.50\left(\mathrm{mg} \cdot \mathrm{g}^{-1} \cdot \mathrm{dw}, \text { HPLC-DAD) [92] }\right.\end{array}$ & - & - & - & - \\
\hline cis-Caftaric acid & - & $\begin{array}{l}\leq 1.30\left(\mathrm{mg} \cdot \mathrm{kg}^{-1} \cdot \mathrm{fw}, \text { HPLC-FD) [84]; }\right. \\
\leq 2.50\left(\mathrm{mg} \cdot \mathrm{g}^{-1} \cdot \mathrm{dw}, \text { HPLC-UV) [82] }\right.\end{array}$ & - & - & - \\
\hline trans-Caftaric acid & $0.05-12.20\left(\mathrm{mg} \cdot \mathrm{g}^{-1} \cdot \mathrm{dw}, \mathrm{HPLC}-\mathrm{DAD}\right)[65,81]$ & $\begin{array}{l}\left.\text { 2.40-31.00 (mg.kg } \mathrm{kg}^{-1} \cdot \mathrm{fw}, \mathrm{HPLC}-\mathrm{FD}\right)[84] \\
\leq 4.00\left(\mathrm{mg} \cdot \mathrm{g}^{-1} \cdot \mathrm{dw}, \mathrm{HPLC}-\mathrm{UV}\right)[82]\end{array}$ & - & - & $18.81-83.36\left(\mathrm{mg} \cdot \mathrm{g}^{-1} \cdot \mathrm{dw}\right.$, HPLC-DAD) [59] \\
\hline$p$-Coumaric acid & $\begin{array}{l}0.01-0.08\left(\mu \mathrm{g} \cdot \mathrm{g}^{-1} \mathrm{dw}, \text { HPLC-DAD) [81]; }\right. \\
0.02-0.03\left(\mathrm{mg} \cdot \mathrm{g}^{-1} \cdot \mathrm{dw}, \text { HPLC-DAD) [92] }\right.\end{array}$ & $\leq 2.0\left(\mathrm{mg} \cdot \mathrm{g}^{-1} \cdot \mathrm{dw}, \mathrm{HPLC}-\mathrm{UV}\right)[82]$ & - & $0.03\left(\mathrm{mg} \cdot \mathrm{g}^{-1} \cdot \mathrm{dw}, \mathrm{HPLC}-\mathrm{DAD}\right)[94]$ & - \\
\hline $\begin{array}{l}\text { trans-Coumaroyltartaric } \\
\text { acid }\end{array}$ & - & - & - & - & $2.12-10.71\left(\mathrm{mg} \cdot \mathrm{g}^{-1} \cdot \mathrm{dw}, \mathrm{HPLC}-\mathrm{DAD}\right)[59]$ \\
\hline Coutaric acid- $O$-hexoside & - & $2.27\left(\mathrm{mg} \cdot \mathrm{g}^{-1} \cdot \mathrm{dw}, \mathrm{HPLC}-\mathrm{UV}\right)[82]$ & - & - & - \\
\hline cis-Coutaric acid & - & $\begin{array}{l}1.60-5.90\left(\mathrm{mg} \cdot \mathrm{kg}^{-1} \cdot \mathrm{fw}, \mathrm{HPLC}-\mathrm{FD}\right)[84] \\
\leq 6.20\left(\mathrm{mg} \cdot \mathrm{g}^{-1} \cdot \mathrm{dw}, \mathrm{HPLC}-\mathrm{UV}\right)[82]\end{array}$ & - & - & - \\
\hline trans-Coutaric acid & - & $\begin{array}{l}1.90-18.00\left(\mathrm{mg} \cdot \mathrm{kg}^{-1} \cdot \mathrm{fw}, \text { HPLC-FD) [84]; }\right. \\
\leq 0.30\left(\mathrm{mg} \cdot \mathrm{g}^{-1} \cdot \mathrm{dw}, \mathrm{HPLC}-\mathrm{UV}\right)[82]\end{array}$ & - & - & - \\
\hline trans-Fertaric acid & - & $\begin{array}{l}\leq 2.60\left(\mathrm{mg} \cdot \mathrm{kg}^{-1} \cdot \mathrm{fw}, \text { HPLC-FD) [84]; }\right. \\
\leq 1.70\left(\mathrm{mg} \mathrm{g}^{-1} \cdot \mathrm{dw}, \text { HPLC-UV) [822] }\right.\end{array}$ & - & - & - \\
\hline trans-Ferulic acid & - & - & - & $0.04\left(\mathrm{mg} \cdot \mathrm{g}^{-1} \cdot \mathrm{dw}, \mathrm{HPLC}-\mathrm{DAD}\right)[94]$ & - \\
\hline
\end{tabular}

HPLC-DAD: High Performance Liquid Chromatography-Diode Array Detector; HPLC-FD: High Performance Liquid Chromatography-Fluorescence Detector; HPLC-UV: High Performance Liquid Chromatography-Ultraviolet Detector; dw, dry weight; fw, fresh weight. 


\subsubsection{Flavonols}

Flavonols are flavonoids characterized by the presence of a double bond between carbons $\mathrm{C} 2$ and C3, and a hydroxyl group in C3 [95]. Different sugars can be bound to the flavonols, producing glycosides, glucuronides, galactosides, and diglycosides (glucosylarabinoside, glucosylgalactoside, glucosylxyloside and glucosylrhamnoside) [96]. Flavonols can react with anthocyanins to produce more stable pigments, and with phenolic acids to participate in copigmentation phenomena $[7,84]$.

The distribution of flavonols in the separate materials of vinification wastes (stems, seeds, skins, pomace, and leaves), according to the bibliography, show differences concerning the individual flavonols as well as to their relative proportions (Tables 3 and 4) and, in general, those materials from red varieties present higher amounts of these compounds than white variety byproducts.

When analyzing the concentration of individual flavonols in red grape stems, it has been observed that a wide variety of flavonols, with remarkable contents of quercetin derivatives, are mainly represented by quercetin-3-O-glucuronide (up to $128 \mathrm{mg} \cdot \mathrm{g}^{-1} \cdot \mathrm{dw}$ ) $[8,45]$. Other major flavonols described in grape stems are quercetin-3-O-glucoside, quercetin-3-O-galactoside, and quercetin-3-Orutinoside $[8,81]$. The stems from red and white varieties showed a similar profile of flavonols, but the plant material from white varieties present a much lower concentration of compounds. The phytochemical profile of grape pomace flavonols is quite simple, with mainly quercetin-3-Oglucuronide being the predominant compound [97-99] (Table 3). The flavonol profile of the constituents of grape pomace as a whole (seeds, skins and some pulp) provides evidenceof higher amounts of phenolics than when considering the separate materials (mainly skins and seeds). Actually, in grape skins, the preponderance of quercetin-3-O-glucuronide (39.5 and $37.5 \mathrm{mg} \cdot \mathrm{kg}^{-1} \cdot \mathrm{fw}$ (fresh weight) in white and red grape skins, respectively) and quercetin-3-O-glucoside (44.0 and $43.0 \mathrm{mg} \cdot \mathrm{kg}^{-1} \cdot \mathrm{fw}$, on average, in white and red grape skins, respectively), remain at similar high levels [100], and interestingly, to the best of our knowledge, no quantitative analysis of quercetin-3-O-galactoside has been performed on grape seeds from red varieties whereas seeds from white cultivars reported contents of this compound. The lack of data on the grape seeds content in flavonols support the idea of an insignificant contribution to their concentration in pomace in addition to the amounts in pulp or stems.

The presence of the flavanonols engeletin $\left(\mathrm{C}_{21} \mathrm{H}_{22} \mathrm{O}_{10}\right)$, as well as the flavonols myricetin $\left(\mathrm{C}_{15} \mathrm{H}_{10} \mathrm{O}_{8}\right)$ and laricitrin $\left(\mathrm{C}_{16} \mathrm{H}_{12} \mathrm{O}_{8}\right)$ has been not detected in organic residues from white varieties. This could be due to the absence of the enzyme flavonoid-3',5'-hydroxylase in white grape varieties $[90,96]$. Concerning "3-O-glycosides" flavonol derivatives in red grapes, they include three different complete series according to the nature of the sugar moiety linked to the $\mathrm{C} 3$ position. The "3-O-glucosides" are the main derivative of the six flavonol aglycones (kaempferol, quercetin, isorhamnetin, myricetin, laricitrin, and syringetin), whereas the correspondent "3-O-galactoside" derivatives are found to be minor compounds. The "3-O-glucuronides" have been described as the third kind of red grapes' flavonol derivatives, and normally account as minor compounds for all of the flavonol aglycones, with the exception of quercetin-3-O-glucuronide, which is as abundant as quercetin 3-O-glucoside [86,101]. 
Table 3. Content in flavan-3-ols and tannins, flavones, and flavonols in winery residues of red varieties of Vitis vinifera L.

\begin{tabular}{|c|c|c|c|c|c|c|}
\hline Compound & Stem & Skin & Seed & Pomace & Leaves & Lees \\
\hline \multicolumn{7}{|c|}{ Flavan-3-ols and tannins } \\
\hline Catechin $^{\mathrm{Z}}$ & $\begin{array}{l}0.71-85.80\left(\mathrm{mg} \cdot \mathrm{g}^{-1} \cdot \mathrm{dw},\right. \\
\text { HPLC-DAD } \\
1.24-2.58\left(\mu \mathrm{g} \cdot \mathrm{g}^{-1} \cdot \mathrm{dw} ; \mathrm{dw} \text { HPLC-DAD) [92] }\right. \\
\leq 60.00\left(\mathrm{mg} \cdot \mathrm{kg}^{-1} \cdot \mathrm{fw}, \mathrm{HPLC}-\mathrm{DAD}\right)[44] \\
0.12-1.27 \mathrm{mg} \cdot \mathrm{g}^{-1} \cdot \mathrm{dw}, \text { HPLC-UV) [103] }\end{array}$ & $\begin{array}{l}8.50-25.0\left(\mathrm{mg} \cdot \mathrm{kg}^{-1} \cdot \mathrm{fw}\right. \\
\text { HPLC-FD) } \\
\leq 13.20\left(\mathrm{mg} \cdot \mathrm{g}^{-1} \cdot \mathrm{dw}\right. \\
\text { HPLC-DAD) }[32] \\
0.01-10.00\left(\mathrm{mg} \cdot \mathrm{g}^{-1} \cdot \mathrm{dw}\right. \\
\text { HPLC-FD) }[104]\end{array}$ & $\begin{array}{l}0.08-4.50\left(\mathrm{mg} \cdot \mathrm{g}^{-1} \cdot \mathrm{fw}\right. \\
\text { HPLC-FD) }[85,105] \\
0.27-1.17\left(\mathrm{mg} \cdot \mathrm{g}^{-1} \cdot \mathrm{dw}\right. \\
\text { HPLC-DAD) }[33]\end{array}$ & $\begin{array}{l}1.12-1.50\left(\mathrm{mg} \cdot \mathrm{g}^{-1} \cdot \mathrm{dw},\right. \\
\text { HPLC-DAD) }[97]\end{array}$ & $71.00\left(\mathrm{mg} \cdot \mathrm{kg}^{-1} \cdot \mathrm{fw}\right.$, HPLC-UV) [86] & - \\
\hline Epicatechin & $\begin{array}{l}\leq 1.00-13.30\left(\mathrm{mg} \cdot \mathrm{g}^{-1} \cdot \mathrm{dw}\right. \\
\text { HPLC-DAD) }[4,61,78,106] \\
\leq 0.11\left(\mathrm{mg} \cdot \mathrm{g}^{-1} \cdot \mathrm{dw}, \mathrm{HPLC}-\mathrm{UV}\right)[103]\end{array}$ & $\begin{array}{l}6.20-13.00\left(\mathrm{mg} \cdot \mathrm{kg}^{-1} \cdot \mathrm{fw}\right. \\
\mathrm{HPLC}-\mathrm{FD})[84] \\
\leq 1.10\left(\mathrm{mg} \cdot \mathrm{g}^{-1} \cdot \mathrm{dw}\right. \\
\text { HPLC-FD) }[104]\end{array}$ & $\begin{array}{l}0.06-0.21\left(\mathrm{mg} \cdot \mathrm{g}^{-1} \cdot \mathrm{fw},\right. \\
\text { HPLC-FD) }[85,105] \\
\leq 0.47\left(\mathrm{mg} \cdot \mathrm{g}^{-1} \cdot \mathrm{dw},\right. \\
\text { HPLC-DAD) [33] }\end{array}$ & $\leq 2.01\left(\mathrm{mg} \cdot \mathrm{g}^{-1} \cdot \mathrm{dw}, \mathrm{HPLC}-\mathrm{UV}\right)[17]$ & $15.00\left(\mathrm{mg} \cdot \mathrm{kg}^{-1} \cdot \mathrm{fw}\right.$, HPLC-UV) [86] & - \\
\hline $\begin{array}{l}\text { Epicatechin } \\
\text { gallate }\end{array}$ & $\begin{array}{l}0.06-7.80\left(\mathrm{mg} \cdot \mathrm{g}^{-1} \cdot \mathrm{dw}, \text { HPLC-DAD) }[65,81]\right. \\
15.50-19.80\left(\mathrm{mg} \cdot \mathrm{kg}^{-1} \cdot \mathrm{fw}, \mathrm{HPLC}-\mathrm{FD}\right)[44]\end{array}$ & - & $\begin{array}{l}13.00-70.00\left(\mathrm{mg} \cdot \mathrm{kg}^{-1} \cdot \mathrm{fw}\right. \\
\text { HPLC-FD) }[85]\end{array}$ & - & $15.00\left(\mathrm{mg} \cdot \mathrm{kg}^{-1} \cdot \mathrm{fw}, \mathrm{HPLC}-\mathrm{UV}\right)[86]$ & - \\
\hline Epigallocatechin & $1.50-5.40\left(\mathrm{mg} \cdot \mathrm{kg}^{-1} \cdot \mathrm{fw}, \mathrm{HPLC}-\mathrm{DAD}\right)[44]$ & - & - & - & - & - \\
\hline Procyanidin B1 & $\begin{array}{l}6.20-13.73\left(\mathrm{mg} \cdot \mathrm{g}^{-1} \cdot \mathrm{dw}, \text { HPLC-DAD) [102] }\right. \\
0.25-1.96\left(\mathrm{mg} \cdot \mathrm{g}^{-1} \cdot \mathrm{dw}, \text { HPLC-UV) [103] }\right.\end{array}$ & $\begin{array}{l}8.40-22.00\left(\mathrm{mg} \cdot \mathrm{kg}^{-1} \cdot \mathrm{fw}\right. \\
\text { HPLC-FD) }[82]\end{array}$ & $\begin{array}{l}74.00-170.00\left(\mathrm{mg} \cdot \mathrm{kg}^{-1} \cdot \mathrm{fw},\right. \\
\text { HPLC-FD) }[85]\end{array}$ & - & - & - \\
\hline Procyanidin B2 & $\begin{array}{l}0.11-5.10\left(\mathrm{mg} \cdot \mathrm{g}^{-1} \cdot \mathrm{dw}, \text { HPLC-DAD) }\right. \\
\leq 0.09\left(\mathrm{mg} \cdot \mathrm{g}^{-1} \cdot \mathrm{dw}, \mathrm{dw}, \mathrm{HPLC}-\mathrm{UV}\right)[103]\end{array}$ & $\begin{array}{l}0.70-2.20\left(\mathrm{mg} \cdot \mathrm{kg}^{-1} \cdot \mathrm{fw}\right. \\
\text { HPLC-FD) }[82]\end{array}$ & $\begin{array}{l}21.00-41.00\left(\mathrm{mg} \cdot \mathrm{kg}^{-1} \cdot \mathrm{fw}\right. \\
\text { HPLC-FD) }[85]\end{array}$ & - & - & - \\
\hline Procyanidin B3 & $\begin{array}{l}0.14-20.50\left(\mathrm{mg} \cdot \mathrm{g}^{-1} \cdot \mathrm{dw}, \text { HPLC-DAD) }[65,81]\right. \\
0.04-0.23\left(\mathrm{mg} \cdot \mathrm{g}^{-1} \cdot \mathrm{dw}, \text { HPLC-UV) }[103]\right.\end{array}$ & $\begin{array}{l}16.00-39.00\left(\mathrm{mg} \cdot \mathrm{kg}^{-1} \cdot \mathrm{fw}\right. \\
\text { HPLC-FD) }\end{array}$ & $\begin{array}{l}43.00-64.00\left(\mathrm{mg} \cdot \mathrm{kg}^{-1} \cdot \mathrm{fw}\right. \\
\text { HPLC-FD) }\end{array}$ & - & - & - \\
\hline Procyanidin B4 & - & - & $\begin{array}{l}33.00-80.00\left(\mathrm{mg} \cdot \mathrm{kg}^{-1} \cdot \mathrm{fw}\right. \\
\text { HPLC-FD) }\end{array}$ & - & - & - \\
\hline $\begin{array}{l}\text { Procyanidin } \\
\text { trimmer } \mathrm{C} 1\end{array}$ & - & $\begin{array}{l}\leq 0.04\left(\mathrm{mg} \cdot \mathrm{g}^{-1} \cdot \mathrm{dw}\right. \\
\text { HPLC-FD) }[104]\end{array}$ & $\begin{array}{l}0.20-0.30\left(\mathrm{mg} \cdot \mathrm{g}^{-1} \cdot \mathrm{dw},\right. \\
\text { HPLC-FD) }[105]\end{array}$ & - & - & - \\
\hline \multicolumn{7}{|l|}{ Flavones } \\
\hline Luteolin & $0.02-0.04\left(\mu \mathrm{g} \cdot \mathrm{g}^{-1} \cdot \mathrm{dw} ;\right.$ HPLC-DAD) [92] & - & - & - & - & - \\
\hline Flavonols & & & & & & \\
\hline $\begin{array}{l}\text { Engeletin } \\
\text { Isorhamnetin }\end{array}$ & Traces (HPLC-DAD) [44] & $\begin{array}{l}- \\
-\end{array}$ & $\begin{array}{l}- \\
-\end{array}$ & $\begin{array}{l}- \\
<0.20\left(\mathrm{mg} \cdot \mathrm{g}^{-1} \cdot \mathrm{dw}, \mathrm{HPLC}-\mathrm{DAD}\right)[97]\end{array}$ & - & - \\
\hline Isrhm-3-O-Glc & - & $\begin{array}{l}11.00-48.00\left(\mathrm{mg} \cdot \mathrm{kg}^{-1} \cdot \mathrm{fw}\right. \\
\text { HPLC-FD) }\end{array}$ & - & $\begin{array}{l}0.06\left(\mathrm{mg} \cdot \mathrm{g}^{-1} \cdot \mathrm{dw}, \text { HPLC-DAD) [96] }\right. \\
\leq 0.10\left(\mathrm{mg} \cdot \mathrm{g}^{-1} \cdot \mathrm{dw}, \text { HPLC-DAD) [97] }\right.\end{array}$ & - & - \\
\hline Isrhm-3-O-Gluc & - & - & - & $\leq 0.90\left(\mathrm{mg} \cdot \mathrm{g}^{-1} \cdot \mathrm{dw}\right.$, HPLC-DAD) [97] & - & - \\
\hline Kaempferol & $\leq 1.80\left(\mathrm{mg} \cdot \mathrm{g}^{-1} \cdot \mathrm{dw}, \mathrm{HPLC}-\mathrm{DAD}\right)[81]$ & - & - & $\begin{array}{l}<0.01\left(\mathrm{mg} \cdot \mathrm{g}^{-1} \cdot \mathrm{dw}, \text { HPLC-DAD) [98] }\right. \\
\leq 0.01\left(\mathrm{mg} \cdot \mathrm{g}^{-1} \cdot \mathrm{dw}, \text { HPLC-DAD) [97] }\right.\end{array}$ & - & - \\
\hline $\mathrm{K}-3-O-\mathrm{Glc}$ & Traces (HPLC-DAD) [44] & $\begin{array}{l}8.00-14.00\left(\mathrm{mg} \cdot \mathrm{kg}^{-1} \cdot \mathrm{fw}\right. \\
\text { HPLC-FD) [84] }\end{array}$ & - & $\begin{array}{l}0.01\left(\mathrm{mg} \cdot \mathrm{g}^{-1} \cdot \mathrm{dw}, \text { HPLC-DAD) [98] }\right. \\
\leq 0.20\left(\mathrm{mg} \cdot \mathrm{g}^{-1} \cdot \mathrm{dw}, \text { HPLC-DAD) [97] }\right.\end{array}$ & $\begin{array}{l}\leq 30.0\left(\mathrm{mg} \cdot \mathrm{kg}^{-1} \cdot \mathrm{fw}, \mathrm{HPLC}-\mathrm{UV}\right)[86] \\
2.03-5.51 \mathrm{mg} \cdot \mathrm{g}^{-1} \cdot \mathrm{dw} \\
\text { HPLC-DAD) [59] }\end{array}$ & - \\
\hline $\begin{array}{l}\text { K-3-O-Gluc } \\
\text { Laricitin }\end{array}$ & Traces (HPLC-DAD) [44] & - & - & $\begin{array}{l}- \\
\leq 0.20\left(\mathrm{mg} \cdot \mathrm{g}^{-1} \cdot \mathrm{dw}, \text { HPLC-DAD)[97] }\right.\end{array}$ & - & - \\
\hline Myricetin & - & - & - & - & - & $\begin{array}{l}4292\left(\mu \mathrm{g} \cdot \mathrm{g}^{-1} \cdot \mathrm{dw}\right. \\
\text { HPLC-DAD) [61] }\end{array}$ \\
\hline Myr-3-O-Glc & Traces (HPLC-DAD) [44] & $\begin{array}{l}13.00-26.00\left(\mathrm{mg} \cdot \mathrm{kg}^{-1} \cdot \mathrm{fw}\right. \\
\text { HPLC-FD) } \\
84]\end{array}$ & - & & $\begin{array}{l}0.49-1.49\left(\mathrm{mg} \cdot \mathrm{g}^{-1} \cdot \mathrm{dw}\right. \\
\text { HPLC-DAD) }[59]\end{array}$ & - \\
\hline Myr-3-O-Gluc & Traces (HPLC-DAD) [44] & $\begin{array}{l}5.80-10.00\left(\mathrm{mg} \cdot \mathrm{kg}^{-1} \cdot \mathrm{fw}\right. \\
\text { HPLC-FD) [84] }\end{array}$ & - & - & - & - \\
\hline Quercetin & $0.60-8.20\left(\mu \mathrm{g} \cdot \mathrm{g}^{-1} \cdot \mathrm{dw}, \mathrm{HPLC}-\mathrm{DAD}\right)[81]$ & - & - & $\begin{array}{l}15.30\left(\mathrm{mg} \cdot \mathrm{kg}^{-1} \cdot \mathrm{dw}, \text { HPLC-DAD) }[97]\right. \\
0.50-2.80\left(\mathrm{mg} \cdot 100 \mathrm{~g}^{-1} \cdot \mathrm{dw},\right. \\
\text { HPLC-DAD) [97] }\end{array}$ & $47.00\left(\mathrm{mg} \cdot \mathrm{kg}^{-1} \cdot \mathrm{fw}, \mathrm{HPLC}-\mathrm{UV}\right)[86]$ & $\begin{array}{l}13,656\left(\mu \mathrm{g} \cdot \mathrm{g}^{-1} \cdot \mathrm{dw}\right. \\
\text { HPLC-DAD) [61] }\end{array}$ \\
\hline Q-3-O-Gal & $6.60-15.00\left(\mathrm{mg} \cdot \mathrm{g}^{-1} \cdot \mathrm{dw}\right.$, HPLC-DAD) [81] & - & - & - & - & - \\
\hline
\end{tabular}


Table 3. Cont.

\begin{tabular}{|c|c|c|c|c|c|c|}
\hline Compound & Stem & Skin & Seed & Pomace & Leaves & Lees \\
\hline Q-3-O-Glc & $\begin{array}{l}0.28-7.30\left(\mathrm{mg} \cdot \mathrm{g}^{-1} \cdot \mathrm{dw},\right. \\
\mathrm{HPLC}-\mathrm{DAD})[81,102] \\
18.0\left(\mathrm{mg} \cdot \mathrm{kg}^{-1} \cdot \mathrm{fw}, \mathrm{HPLC}-\mathrm{DAD}\right)[44]\end{array}$ & $\begin{array}{l}31.00-55.00\left(\mathrm{mg} \cdot \mathrm{kg}^{-1} \cdot \mathrm{fw},\right. \\
\text { HPLC-FD) [84] }\end{array}$ & - & $\begin{array}{l}0.18\left(\mathrm{mg} \cdot \mathrm{g}^{-1} \cdot \mathrm{dw}, \mathrm{HPLC}-\mathrm{DAD}\right)[98] \\
\leq 0.90-5.1\left(\mathrm{mg} \cdot \mathrm{g}^{-1} \cdot \mathrm{dw},\right. \\
\text { HPLC-DAD) [97,99] }\end{array}$ & $142.00\left(\mathrm{mg} \cdot \mathrm{kg}^{-1} \cdot \mathrm{fw}, \mathrm{HPLC}-\mathrm{UV}\right)[86]$ & - \\
\hline Q-3-O-GlcXyl & - & $\begin{array}{l}9.00-18.00\left(\mathrm{mg} \cdot \mathrm{kg}^{-1} \cdot \mathrm{fw}\right. \\
\text { HPLC-FD) }[84]\end{array}$ & - & - & - & - \\
\hline Q-3-O-Gluc & $\begin{array}{l}0.20-126.80 \mathrm{mg} \cdot \mathrm{g}^{-1} \cdot \mathrm{dw} \\
\text { HPLC-DAD) }[44,102]\end{array}$ & $\begin{array}{l}29.00-59.00\left(\mathrm{mg} \cdot \mathrm{kg}^{-1} \cdot \mathrm{fw},\right. \\
\text { HPLC-FD) [84] }\end{array}$ & - & $\begin{array}{l}\leq 1.30-130.00\left(\mathrm{mg} \cdot \mathrm{g}^{-1} \cdot \mathrm{dw},\right. \\
\text { HPLC-DAD) }[97,98] \\
\leq 105.40\left(\mathrm{mg} \cdot \mathrm{g}^{-1} \cdot \mathrm{dw}, \mathrm{HPLC}-\mathrm{DAD}\right)[99]\end{array}$ & - & $\begin{array}{l}8900\left(\mu \mathrm{g} \cdot \mathrm{g}^{-1} \cdot \mathrm{dw},\right. \\
\text { HPLC-DAD) [61] }\end{array}$ \\
\hline Q-3-O-Rha & $0.30-2.80\left(\mathrm{mg} \cdot \mathrm{g}^{-1} \cdot \mathrm{dw}, \mathrm{HPLC}-\mathrm{DAD}\right)[81]$ & - & - & & - & - \\
\hline Q-3-O-Rut & $1.90-41.80\left(\mathrm{mg} \cdot \mathrm{g}^{-1} \cdot \mathrm{dw}\right.$, HPLC-DAD) [81] & $\begin{array}{l}0.01-0.57\left(\mathrm{mg} \cdot \mathrm{g}^{-1} \cdot \mathrm{dw},\right. \\
\text { HPLCDADD [32,99] }\end{array}$ & - & $\begin{array}{l}0.12-0.50\left(\mathrm{mg} \cdot \mathrm{g}^{-1} \cdot \mathrm{dw},\right. \\
\text { HPLC-DAD) }[33,97]\end{array}$ & - & - \\
\hline $\begin{array}{l}\text { Flavanonols } \\
\text { Astilbin }\end{array}$ & $35.00\left(\mathrm{HPLC}-\mathrm{DAD} ; \mathrm{mg} \cdot \mathrm{kg}^{-1} \cdot \mathrm{fw}\right)$ [44] & - & - & - & - & - \\
\hline
\end{tabular}

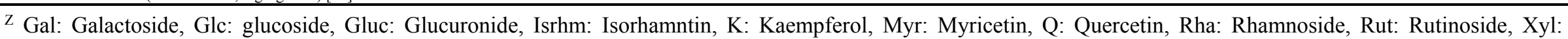

Xyloside; Y HPLC-DAD: High Performance Liquid Chromatography-Diode Array Detector, HPLC-FD: High Performance Liquid Chromatography-Fluorescence

Detector, HPLC-UV: High Performance Liquid Chromatography-Ultraviolet Detector; dw, dry weight; fw, fresh weight.

Table 4. Content in flavan-3-ols and tannins, flavones, and flavonols in winery residues of white varieties of Vitis vinifera L.

\begin{tabular}{|c|c|c|c|c|c|}
\hline Compound & Stem & Skin & Seed & Pomace & Leaves \\
\hline \multicolumn{6}{|l|}{ Flavan-3-ols and tannins } \\
\hline \multirow{4}{*}{ Catechin ${ }^{z}$} & $46.50-98.30\left(\mu \mathrm{g} \cdot \mathrm{g}^{-1} \cdot \mathrm{dw}\right.$, HPLC-DAD $\left.{ }^{\mathrm{Y}}\right)[84]$ & & $120.00-500.00\left(\mathrm{mg} \cdot \mathrm{kg}^{-1} \cdot \mathrm{fw}\right.$, & \multirow{4}{*}{$<0.30\left(\mathrm{mg} \cdot \mathrm{g}^{-1} \cdot \mathrm{dw}, \mathrm{HPLC}-\mathrm{DAD}\right)[108,109]$} & \multirow{4}{*}{ - } \\
\hline & $0.13-2.89\left(\mathrm{mg} \cdot \mathrm{g}^{-1} \cdot \mathrm{dw}\right.$, HPLC-DAD) $[92,93]$ & 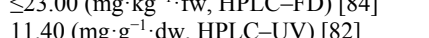 & $\begin{array}{l}\text { HPLC-FD) [84] } \\
106.50\left(\mathrm{mg} \cdot \mathrm{g}^{-1} \cdot \mathrm{dw}, \mathrm{HPLC}-\mathrm{UV}\right)[82]\end{array}$ & & \\
\hline & $3.85-18.58\left(\mu \mathrm{g} \cdot \mathrm{g}^{-1} \cdot \mathrm{dw} ; \mathrm{HPLC}-\mathrm{DAD}\right)[65]$ & $0.72-0.84\left(\mathrm{mg} \cdot \mathrm{g}^{-1} \cdot \mathrm{dw}\right.$, HPLC-DAD) [93] & $83.1-98.3\left(\mathrm{mg} \cdot \mathrm{g}^{-1} \cdot \mathrm{dw}, \mathrm{HPLC}-\mathrm{UV}\right)[107]$ & & \\
\hline & $9.30-133.90\left(\mathrm{mg} \cdot \mathrm{g}^{-1} \cdot \mathrm{dw}, \mathrm{HPLC}-\mathrm{UV}\right)[103]$ & & $0.45-0.53\left(\mathrm{mg} \cdot \mathrm{g}^{-1} \cdot \mathrm{dw}, \mathrm{HPLC}-\mathrm{DAD}\right)[93]$ & & \\
\hline \multirow[t]{2}{*}{ Dimer gallate } & - & - & $38.1-46.8\left(\mathrm{mg} \cdot \mathrm{g}^{-1} \cdot \mathrm{dw}\right.$, HPLC-UV) [107] & \multirow[t]{2}{*}{ - } & \multirow[t]{2}{*}{ - } \\
\hline & $\leq 4.00-0.58\left(\mu \mathrm{g} \cdot \mathrm{g}^{-1} \cdot \mathrm{dw}, \mathrm{HPLC}-\mathrm{DAD}\right)[65,81]$ & $\leq 8.30\left(\mathrm{mg} \cdot \mathrm{kg}^{-1} \cdot \mathrm{fw}, \mathrm{HPLC}-\mathrm{FD}\right)[84]$ & $\begin{array}{l}110.00-310.00\left(\mathrm{mg} \cdot \mathrm{kg}^{-1} \cdot \mathrm{fw},\right. \\
\text { HPLC-FD) [84] }\end{array}$ & & \\
\hline \multirow[t]{2}{*}{ Epicatechin } & $0.04-1.13\left(\mathrm{mg} \cdot \mathrm{g}^{-1} \cdot \mathrm{dw}\right.$, HPLC-DAD) $[93,110]$ & $2.70\left(\mathrm{mg} \cdot \mathrm{g}^{-1} \cdot \mathrm{dw}, \mathrm{HPLC}-\mathrm{UV}\right)[82]$ & $77.50\left(\mathrm{mg} \cdot \mathrm{g}^{-1} \cdot \mathrm{dw}, \mathrm{HPLC}-\mathrm{UV}\right)[82]$ & \multirow[t]{2}{*}{$<0.07\left(\mathrm{mg} \cdot \mathrm{g}^{-1} \cdot \mathrm{dw}\right.$, HPLC-DAD) [108] } & \multirow[t]{2}{*}{ - } \\
\hline & $0.50-5.80\left(\mathrm{mg} \cdot \mathrm{g}^{-1} \cdot \mathrm{dw}, \mathrm{HPLC}-\mathrm{UV}\right)[103]$ & $0.77-0.85\left(\mathrm{mg} \cdot \mathrm{g}^{-1} \cdot \mathrm{dw}\right.$, HPLC-DAD) [93] & $\begin{array}{l}54.2-55.8\left(\mathrm{mg} \cdot \mathrm{g}^{-1} \cdot \mathrm{dw}, \text { HPLC-UV) [107] }\right. \\
0.79-0.89\left(\mathrm{mg} \cdot \mathrm{g}^{-1} \cdot \mathrm{dw}, \mathrm{HPLC}-\mathrm{DAD}\right)[107]\end{array}$ & & \\
\hline Epicatechin-epicatechingallate I & - & - & $26.80\left(\mathrm{mg}^{-\mathrm{g}^{-1}} \cdot \mathrm{dw}, \mathrm{HPLC}-\mathrm{UV}\right)[82]$ & _ & _- \\
\hline Epicatechin-epicatechingallate II & - & - & $23.70\left(\mathrm{mg} \cdot \mathrm{g}^{-1} \cdot \mathrm{dw}, \mathrm{HPLC}-\mathrm{UV}\right)[82]$ & - & - \\
\hline Epicatechin-epicatechingallate III & - & - & $21.40\left(\mathrm{mg}^{-\mathrm{g}^{-1}} \cdot \mathrm{dw}, \mathrm{HPLC}-\mathrm{UV}\right)[82]$ & - & - \\
\hline Epicatechin gallate & $0.34-15.70\left(\mu \mathrm{g} \cdot \mathrm{g}^{-1} \cdot \mathrm{dw}\right.$, HPLC-DAD) $[44,65,81]$ & - & $\begin{array}{l}13.00-67.00\left(\mathrm{mg} \cdot \mathrm{kg}^{-1} \cdot \mathrm{fw}, \text { HPLC-FD) [84] }\right. \\
76.50\left(\mathrm{mg} \cdot \mathrm{g}^{-1} \cdot \mathrm{dw}, \mathrm{HPLC}-\mathrm{UV}\right)[82]\end{array}$ & $<0.05\left(\mathrm{mg} \cdot \mathrm{g}^{-1} \cdot \mathrm{dw}\right.$, HPLC-DAD) [108] & - \\
\hline Epicatechin-3-hexose & - & _- & $1.50\left(\mathrm{mg} \cdot \mathrm{g}^{-1} \cdot \mathrm{dw}\right.$, HPLC-UV) [82] & _ & _- \\
\hline Epigallocatechin & $0.80-0.90\left(\mathrm{mg} \cdot \mathrm{kg}^{-1} \cdot \mathrm{fw}, \mathrm{HPLC}-\mathrm{DAD}\right)[44]$ & $2.10\left(\mathrm{mg} \cdot \mathrm{g}^{-1} \cdot \mathrm{dw}, \mathrm{HPLC}-\mathrm{UV}\right)[82]$ & & - & - \\
\hline Epigallocatechin gallate & 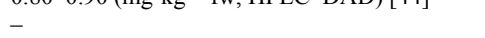 & - & $0.30-0.50\left(\mathrm{mg} \cdot \mathrm{g}^{-1} \cdot \mathrm{dw}, \mathrm{HPLC}-\mathrm{UV}\right)[107]$ & - & - \\
\hline Epigallocatechin-epicatechin & - & - & $3.60\left(\mathrm{mg} \cdot \mathrm{g}^{-1} \cdot \mathrm{dw}, \mathrm{HPLC}-\mathrm{UV}\right)[82]$ & - & - \\
\hline Procyanidin dimmer B1 & $\begin{array}{l}13.30-187.70\left(\mathrm{mg} \cdot \mathrm{g}^{-1} \cdot \mathrm{dw}, \text { HPLC-UV) [103] }\right. \\
0.07-0.11\left(\mathrm{mg} \cdot \mathrm{g}^{-1} \cdot \mathrm{dw}, \text { HPLC-DAD) [93] }\right.\end{array}$ & $\begin{array}{l}\leq 48.00\left(\mathrm{mg} \cdot \mathrm{kg}^{-1} \cdot \mathrm{fw}, \text { HPLC-FD) [84] }\right. \\
0.36-0.48\left(\mathrm{mg} \cdot \mathrm{g}^{-1} \cdot \mathrm{dw}, \text { HPLC-DAD) [93] }\right.\end{array}$ & $\begin{array}{l}200.00-620.00\left(\mathrm{mg} \cdot \mathrm{kg}^{-1} \cdot \mathrm{fw},\right. \\
\text { HPLC-FD) [82] } \\
3.10\left(\mathrm{mg} \cdot \mathrm{g}^{-1} \cdot \mathrm{dw}, \mathrm{HPLC}-\mathrm{UV}\right)[82] \\
0.10-0.14\left(\mathrm{mg} \cdot \mathrm{g}^{-1} \cdot \mathrm{dw}, \mathrm{HPLC}-\mathrm{DAD}\right)[107]\end{array}$ & $<0.22\left(\mathrm{mg} \cdot \mathrm{g}^{-1} \cdot \mathrm{dw}, \mathrm{HPLC}-\mathrm{DAD}\right)[93,108]$ & - \\
\hline
\end{tabular}


Table 4. Cont.

\begin{tabular}{|c|c|c|c|c|c|}
\hline Compound & Stem & Skin & Seed & Pomace & Leaves \\
\hline Procyanidin dimmer B2 & $\begin{array}{l}0.02-8.50\left(\mathrm{mg} \cdot \mathrm{g}^{-1} \cdot \mathrm{dw}, \text { HPLC-DAD) }[65,81,93]\right. \\
1.10-4.80\left(\mathrm{mg} \cdot \mathrm{g}^{-1} \cdot \mathrm{dw}, \text { HPLC-UV) }[103]\right.\end{array}$ & $\begin{array}{l}8.70\left(\mathrm{mg} \cdot \mathrm{g}^{-1} \cdot \mathrm{dw}, \text { HPLC-UV) [82] }\right. \\
0.59-0.65\left(\mathrm{mg} \cdot \mathrm{g}^{-1} \cdot \mathrm{dw}, \text { HPLC-DAD) [93] }\right.\end{array}$ & $\begin{array}{l}15.00-33.00\left(\mathrm{mg} \cdot \mathrm{kg}^{-1} \cdot \mathrm{fw}, \text { HPLC-FD) [84] }\right. \\
64.50\left(\mathrm{mg} \cdot \mathrm{g}^{-1} \cdot \mathrm{dw}, \mathrm{HPLC}-\mathrm{HV}\right)[82] \\
0.79-0.91\left(\mathrm{mg} \cdot \mathrm{g}^{-1} \cdot \mathrm{dw}, \mathrm{HPLC}-\mathrm{DAD}\right)[107]\end{array}$ & $<0.06\left(\mathrm{mg} \cdot \mathrm{g}^{-1} \cdot \mathrm{dw}\right.$, HPLC-DAD) $[93,108]$ & - \\
\hline Procyanidin dimmer B3 & $\begin{array}{l}0.02-31.60\left(\mathrm{mg} \cdot \mathrm{g}^{-1} \cdot \mathrm{dw},\right. \\
\text { HPLC-DAD) [65,81,93] } \\
4.50-22.20 \mathrm{mg} \cdot \mathrm{g}^{-1} \cdot \mathrm{dw} \text { (HPLC-UV) [111] }\end{array}$ & $\begin{array}{l}\leq 37.00\left(\mathrm{mg} \cdot \mathrm{kg}^{-1} \cdot \mathrm{fw}, \text { HPLC-FD) [84] }\right. \\
0.31-0.35\left(\mathrm{mg} \cdot \mathrm{g}^{-1} \cdot \mathrm{dw}, \text { HPLC-DAD) [93] }\right.\end{array}$ & $\begin{array}{l}3900-56.00\left(\mathrm{mg} \cdot \mathrm{kg}^{-1} \cdot \mathrm{fw}, \text { HPLC-FD) [84] }\right. \\
44.60\left(\mathrm{mg} \cdot \mathrm{g}^{-1} \cdot \mathrm{dw}, \mathrm{HPLC}-\mathrm{UV}\right)[82] \\
0.11-0.15\left(\mathrm{mg} \cdot \mathrm{g}^{-1} \cdot \mathrm{dw}, \text { HPLC-DAD) [107] }\right.\end{array}$ & $<0.06\left(\mathrm{mg} \cdot \mathrm{g}^{-1} \cdot \mathrm{dw}\right.$, HPLC-DAD) [108] & - \\
\hline Procyanidin dimmer B4 & $0.03-0.05\left(\mathrm{mg} \cdot \mathrm{g}^{-1} \cdot \mathrm{dw}, \mathrm{HPLC}-\mathrm{DAD}\right)[93]$ & $\begin{array}{l}8.00\left(\mathrm{mg} \cdot \mathrm{g}^{-1} \cdot \mathrm{dw}, \text { HPLC-UV) [82] }\right. \\
0.26-0.30\left(\mathrm{mg} \cdot \mathrm{g}^{-1} \cdot \mathrm{dw}, \text { HPLC-DAD) [93] }\right.\end{array}$ & $\begin{array}{l}40.00-95.00\left(\mathrm{mg} \cdot \mathrm{kg}^{-1} \cdot \mathrm{fw}, \text { HPLC-FD) [84] }\right. \\
58.40\left(\mathrm{mg} \cdot \mathrm{g}^{-1} \cdot \mathrm{dw}, \mathrm{HPLC}-\text { UV) [82] }\right. \\
0.21-0.31\left(\mathrm{mg} \cdot \mathrm{g}^{-1} \cdot \mathrm{dw}, \text { HPLC-DAD) [107] }\right.\end{array}$ & $<0.07$ (mg g g${ }^{-1} \mathrm{dw}$, HPLC-DAD) [108] & - \\
\hline $\begin{array}{l}\text { Procyanidin B1-O-gallate } \\
\text { Procyanidin B2-O-gallate }\end{array}$ & $\begin{array}{l}0.04\left(\mathrm{mg} \cdot \mathrm{g}^{-1} \cdot \mathrm{dw}, \text { HPLC-DAD) [93] }\right. \\
0.02\left(\mathrm{mg} \cdot \mathrm{g}^{-1} \cdot \mathrm{dw}, \text { HPLC-DAD) [93] }\right.\end{array}$ & $\begin{array}{l}0.31-0.35\left(\mathrm{mg} \cdot \mathrm{g}^{-1} \cdot \mathrm{dw}, \text { HPLC-DAD) [93] }\right. \\
0.27-0.30\left(\mathrm{mg} \cdot \mathrm{g}^{-1} \cdot \mathrm{dw}, \mathrm{HPLC}-D A D\right)[93]\end{array}$ & $\begin{array}{l}0.66-0.82\left(\mathrm{mg} \cdot \mathrm{g}^{-1} \cdot \mathrm{dw}, \text { HPLC-DAD) [107] }\right. \\
0.66-0.79\left(\mathrm{mg} \cdot \mathrm{g}^{-1} \cdot \mathrm{dw}, \text { HPLC-DAD) [107] }\right.\end{array}$ & $\begin{array}{l}- \\
<0.14\left(\mathrm{mg} \cdot \mathrm{g}^{-1} \cdot \mathrm{dw}, \mathrm{HPLC}-\mathrm{DAD}\right)[108]\end{array}$ & - \\
\hline Procyanidin trimmer $\mathrm{C} 1$ & $0.11-0.19\left(\mathrm{mg} \cdot \mathrm{g}^{-1} \cdot \mathrm{dw}\right.$, HPLC-DAD) [93] & $0.35\left(\mathrm{mg} \cdot \mathrm{g}^{-1} \cdot \mathrm{dw}, \mathrm{HPLC}-\mathrm{DAD}\right)[93]$ & $\begin{array}{l}12.70-31.40\left(\mathrm{mg} \cdot \mathrm{g}^{-1} \cdot \mathrm{dw}, \text { HPLC-UV) [82] }\right. \\
0.51-0.61\left(\mathrm{mg} \cdot \mathrm{g}^{-1} \cdot \mathrm{dw}, \text { HPLC-DAD) [107] }\right.\end{array}$ & $<0.03\left(\mathrm{mg} \cdot \mathrm{g}^{-1} \cdot \mathrm{dw}, \mathrm{HPLC}-\mathrm{DAD}\right)[108]$ & - \\
\hline Procyanidin trimmer $\mathrm{C} 2$ & - & - & $18.50\left(\mathrm{mg} \cdot \mathrm{g}^{-1} \cdot \mathrm{dw}, \mathrm{HPLC}-\mathrm{UV}\right)[82]$ & $<0.04\left(\mathrm{mg} \cdot \mathrm{g}^{-1} \cdot \mathrm{dw}\right.$, HPLC-DAD) [108] & - \\
\hline Procyanidin trimmer $\mathrm{C} 3$ & - & - & $13.50\left(\mathrm{mg} \cdot \mathrm{g}^{-1} \cdot \mathrm{dw}, \mathrm{HPLC}-\mathrm{UV}\right)[82]$ & & - \\
\hline Galloyled-procyanidin & - & - & - & $<0.07\left(\mathrm{mg} \cdot \mathrm{g}^{-1} \cdot \mathrm{dw}\right.$, HPLC-DAD) [108] & - \\
\hline Gallocatechin-catechin dimer & - & - & - & $<0.03\left(\mathrm{mg} \cdot \mathrm{g}^{-1} \cdot \mathrm{dw}\right.$, HPLC-DAD) $[108]$ & - \\
\hline Procyanidin tetramer & - & - & $1.80-2.20\left(\mathrm{mg} \cdot \mathrm{g}^{-1} \cdot \mathrm{dw}\right.$, HPLC-UV) [107] & $<0.06\left(\mathrm{mg} \cdot \mathrm{g}^{-1} \cdot \mathrm{dw}\right.$, HPLC-DAD) $[108]$ & \\
\hline $\begin{array}{l}\text { Flavones } \\
\text { Luteolin }\end{array}$ & $0.01-0.07\left(\mu \mathrm{g} \cdot \mathrm{g}^{-1} \cdot \mathrm{dw}\right.$, HPLC-DAD) [16] & - & - & - & - \\
\hline Flavonols & & & & & \\
\hline $\begin{array}{l}\text { Isrhm-3-O-Glc } \\
\text { Kaempferol }\end{array}$ & 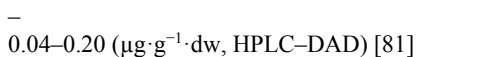 & $\begin{array}{l}\leq 2.30\left(\mathrm{mg} \cdot \mathrm{kg}^{-1} \cdot \mathrm{fw}, \text { HPLC-FD) [84] }\right. \\
3.2\left(\mathrm{mg} \cdot \mathrm{g}^{-1} \cdot \mathrm{dw}, \mathrm{HPLC}-\mathrm{UV}\right)[82]\end{array}$ & - & $<0.01\left(\mathrm{mg} \cdot \mathrm{g}^{-1} \cdot \mathrm{dw}, \mathrm{HPLC}-\mathrm{DAD}\right)[108]$ & - \\
\hline K-3-O-Glc & - & $\begin{array}{l}2.00-26.00\left(\mathrm{mg} \cdot \mathrm{kg}^{-1} \cdot \mathrm{fw}, \text { HPLC-FD) [84] }\right. \\
8.40\left(\mathrm{mg} \cdot \mathrm{g}^{-1} \cdot \mathrm{dw} \text {, HPLC-UV) [82] }\right. \\
0.10\left(\mathrm{mg} \cdot \mathrm{g}^{-1} \cdot \mathrm{dw} \text {. HPLC-UV) [107] }\right.\end{array}$ & - & $<0.02\left(\mathrm{mg} \cdot \mathrm{g}^{-1} \cdot \mathrm{dw}\right.$, HPLC-DAD) $[108]$ & $\begin{array}{l}34.79-62.04\left(\mathrm{mg} \cdot \mathrm{g}^{-1} \cdot \mathrm{dw}\right. \\
\text { HPLC-DAD) }[59]\end{array}$ \\
\hline $\mathrm{K}-3-O$-Gluc & $3.20\left(\mathrm{mg} \cdot \mathrm{g}^{-1} \cdot \mathrm{dw}\right.$, HPLC-UV) [82] & - & - & $<0.01\left(\mathrm{mg} \cdot \mathrm{g}^{-1} \cdot \mathrm{dw}, \mathrm{HPLC}-\mathrm{DAD}\right)[108]$ & - \\
\hline Myr-3-O-Glc & - & - & - & - & $\begin{array}{l}1.11-8.50\left(\mathrm{mg} \cdot \mathrm{g}^{-1} \cdot \mathrm{dw},\right. \\
\text { HPLC-DAD [59] }\end{array}$ \\
\hline $\begin{array}{l}\text { Quercetin } \\
\text { Q-3-O-Gal }\end{array}$ & $\begin{array}{l}0.30-2.20\left(\mu \mathrm{g} \cdot \mathrm{g}^{-1} \cdot \mathrm{dw}, \text { HPLC-DAD) [81] }\right. \\
13.50-19.20\left(\mu \mathrm{g} \cdot \mathrm{g}^{-1} \cdot \mathrm{dw}, \text { HPLC-DAD) [81] }\right.\end{array}$ & $0.30\left(\mathrm{mg} \cdot \mathrm{g}^{-1} \cdot \mathrm{dw}, \mathrm{HPLC}-\mathrm{UV}\right)[107]$ & $\begin{array}{l}- \\
0.20\left(\mathrm{mg} \cdot \mathrm{g}^{-1} \cdot \mathrm{dw}, \mathrm{HPLC}-\mathrm{UV}\right)[107]\end{array}$ & $\begin{array}{l}- \\
<0.02\left(\mathrm{mg} \cdot \mathrm{g}^{-1} \cdot \mathrm{dw}, \mathrm{HPLC}-\mathrm{DAD}\right)[108]\end{array}$ & 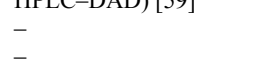 \\
\hline Q-3-O-Glc & $4.50-7.20\left(\mu \mathrm{g} \cdot \mathrm{g}^{-1} \cdot \mathrm{dw}\right.$, HPLC-DAD) [81] & $\begin{array}{l}8.90-66.00\left(\mathrm{mg} \cdot \mathrm{kg}^{-1} \cdot \mathrm{fw}, \mathrm{HPLC}-\mathrm{FD}\right)[84] \\
12.40\left(\mathrm{mg} \cdot \mathrm{g}^{-1} \cdot \mathrm{dw}^{-} \mathrm{HPLC}-\mathrm{HV}\right)[82] \\
12.00-67.00\left(\mathrm{mg} \cdot \mathrm{kg}^{-1} \cdot \mathrm{fw},\right.\end{array}$ & - & $<0.11\left(\mathrm{mg} \cdot \mathrm{g}^{-1} \cdot \mathrm{dw}\right.$, HPLC-DAD) [108] & - \\
\hline Q-3-O-Gluc & - & $\begin{array}{l}\text { HPLC-FD) [84] } \\
1.00\left(\mathrm{mg} \cdot \mathrm{g}^{-1} \cdot \mathrm{dw}, \mathrm{HPLC}-\mathrm{UV}\right)[82]\end{array}$ & - & $<0.09\left(\mathrm{mg} \cdot \mathrm{g}^{-1} \cdot \mathrm{dw}\right.$, HPLC-DAD) [108] & - \\
\hline Q-3-O-pentoside & - & $0.20\left(\mathrm{mg} \cdot \mathrm{g}^{-1} \cdot \mathrm{dw}, \mathrm{HPLC}-\mathrm{UV}\right)[82]$ & - & - & - \\
\hline Q-3-O-Rha & $0.30-1.90\left(\mu \mathrm{g} \cdot \mathrm{g}^{-1} \cdot \mathrm{dw}\right.$, HPLC-DAD) [81] & $-\quad$ & - & - & - \\
\hline Q-3-O-Rut & - & $0.40\left(\mathrm{mg} \cdot \mathrm{g}^{-1} \cdot \mathrm{dw}, \mathrm{HPLC}-\mathrm{UV}\right)[7]$ & - & $<0.02\left(\mathrm{mg} \cdot \mathrm{g}^{-1} \cdot \mathrm{dw}\right.$, HPLC-DAD) $[108]$ & - \\
\hline $\begin{array}{l}\text { Flavanonols } \\
\text { Astilbin }\end{array}$ & - & $5.60\left(\mathrm{mg} \cdot \mathrm{g}^{-1} \cdot \mathrm{dw}, \mathrm{HPLC}-\mathrm{UV}\right)[80]$ & - & - & - \\
\hline
\end{tabular}

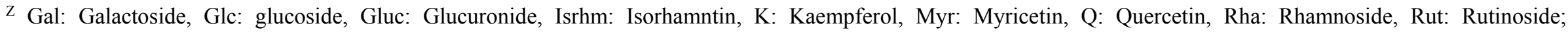

Y HPLC-DAD: High Performance Liquid Chromatography-Diode Array Detector, HPLC-FD: High Performance Liquid Chromatography-Fluorescence Detector,

HPLC-UV: High Performance Liquid Chromatography-Ultraviolet Detector; dw, dry weight; fw, fresh weight. 
Grapevine leaves have been scarcely evaluated on their content in phenolics (Tables 1-7). Actually, the studies available on the flavonol composition of grapevine leaves highlighted the presence of glucoside derivatives in high concentrations, surpassing the content recorded in the other vinification residues (stems, skins, seeds, and pomace) [59,87]. The presence of flavonoids in leaves has been pointed out as a plant physiologic strategy related with their capacity to act as UV filters, protecting some cell structures, like chloroplasts, from harmful effects of UV radiation [112].

\subsubsection{Flavanols}

Flavanols, also called flavan-3-ols, are flavonoid type compounds that have a hydroxyl group at the $\mathrm{C}_{3}$ position and no carbonyl group at the $\mathrm{C}_{4}$ position. These types of flavonoids have been described from the point of view of the physiological role in plants as attractants to pollinators, dyers, and contributors to the organoleptic properties of plant foods. In wineries, flavanols are the major compounds responsible for the astringency, bitterness, and structure of wines [84,113]. On the other hand, tannins are complex phenolic compounds of high molecular weight that can be divided in two distinct groups: condensed tannins and hydrolysable tannins [90]. Condensed tannins, also known as proanthocyanidins, are constituted by subunits of flavan-3-ols monomers and their structures vary depending on their constitutive subunits, the degree of polymerization, and the linkage position [91], whereas hydrolyzable tannins are complex (poly)phenolics that can be degraded into smaller units, mainly sugars and phenolic acids [68].

The evaluation of winery by-products on their content in bioactive compounds as support for further rational applications has allowed to establish the differential concentration of flavan-3-ols and tannins in the separate fractions of vinification by-products (stems, seeds, skins, pomace, and leaves; Tables 3 and 4).

The comparison of concentrations of flavan-3-ols in different grape stems establish that catechin is the major compound in all winery residues from both red and white grapes, and the concentrations in grape stems are higher than those described in skins and seeds. In this regard, the highest concentration of catechins described in red grape stems $\left(85.80 \mathrm{mg} \cdot \mathrm{g}^{-1} \cdot \mathrm{dw}\right.$, on average) exceed those recorded in skins and seeds by up to $85 \%$, on average. Concerning white grapes, in which catechins are also the most abundant flavan-3-ols in all tissues, grape stems are the best source with concentrations higher than the maximal contents described in skins or leaves ( $92 \%$ and $21 \%$ lower, on average, respectively). 
Table 5. Content in anthocyanins of winery residues from different red varieties of Vitis vinifera $\mathrm{L}$.

\begin{tabular}{|c|c|c|c|}
\hline Compound & Skin & Pomace & Lees \\
\hline \multirow{2}{*}{$\mathrm{Cy}-3-O-\mathrm{Glc}^{\mathrm{z}}$} & $\leq 0.04\left(\mathrm{mg} \cdot \mathrm{g}^{-1} \cdot \mathrm{dw}, \mathrm{HPLC}-\mathrm{DAD}^{\mathrm{Y}}\right)[99]$ & $<0.01\left(\mathrm{mg} \cdot \mathrm{g}^{-1} \cdot \mathrm{dw}, \mathrm{HPLC}-\mathrm{UV}-\mathrm{DAD}\right)[97,99]$ & \multirow{2}{*}{-} \\
\hline & $\leq 1.00\left(\mathrm{mg} \cdot \mathrm{g}^{-1} \cdot \mathrm{dw}, \mathrm{HPLC}-\mathrm{FD}\right)[104]$ & $0.01-1.79\left(\mathrm{mg} \cdot \mathrm{g}^{-1} \cdot \mathrm{dw}, \mathrm{HPLC}-\mathrm{DAD}\right)[99]$ & \\
\hline Cy-3-O-(6'-O-acetyl)-Glc & - & $<0.01\left(\mathrm{mg} \cdot \mathrm{g}^{-1} \cdot \mathrm{dw}\right.$, HPLC-UV-DAD) [99] & - \\
\hline \multirow{2}{*}{ Del-3-O-Glc } & $0.02-0.19\left(\mathrm{mg} \cdot \mathrm{g}^{-1} \cdot \mathrm{dw}\right.$, HPLC-DAD) [99] & $\leq 1.20\left(\mathrm{mg} \cdot \mathrm{g}^{-1} \cdot \mathrm{dw}, \mathrm{HPLC}-\mathrm{UV}-\mathrm{DAD}\right)[97,99]$ & \multirow{2}{*}{-} \\
\hline & $\leq 4.2\left(\mathrm{mg} \cdot \mathrm{g}^{-1} \cdot \mathrm{dw}, \mathrm{HPLC}-\mathrm{FD}\right)[104]$ & $0.03-4.24\left(\mathrm{mg} \cdot \mathrm{g}^{-1} \cdot \mathrm{dw}, \mathrm{HPLC}-\mathrm{DAD}\right)[99]$ & \\
\hline Del-3-O-(6'-O-p-coumaryl)-Glc & - & $\leq 2.2\left(\mathrm{mg} \cdot \mathrm{g}^{-1} \cdot \mathrm{dw}, \mathrm{HPLC}-\mathrm{UV}-\mathrm{DAD}\right)[97]$ & - \\
\hline \multirow{2}{*}{ Mv-3-O-Glc } & \multirow{2}{*}{$12.10-16.50\left(\mathrm{mg} \cdot \mathrm{g}^{-1} \cdot \mathrm{dw}\right.$, HPLC-DAD $)[99]$} & $0.06-10.40\left(\mathrm{mg} \cdot \mathrm{g}^{-1} \cdot \mathrm{dw}, \mathrm{HPLC}-\mathrm{UV}-\mathrm{DAD}\right)[97,99]$ & \multirow{2}{*}{$0.091\left(\mathrm{mg} \cdot \mathrm{g}^{-1} \cdot \mathrm{dw}\right.$, HPLC-DAD) [61] } \\
\hline & & $0.095-14.61\left(\mathrm{mg} \cdot \mathrm{g}^{-1} \cdot \mathrm{dw}, \mathrm{HPLC}-\mathrm{DAD}\right)[99]$ & \\
\hline Mv-3-O-(6'-O-acetyl)-Glc & - & $\leq 2.0\left(\mathrm{mg} \cdot \mathrm{g}^{-1} \cdot \mathrm{dw}, \mathrm{HPLC}-\mathrm{UV}-\mathrm{DAD}\right)[97,99]$ & - \\
\hline $\mathrm{Mv}-3-O-(6 '-O$-caffeoyl)-Glc & $29.0-59.0\left(\mathrm{mg} \cdot \mathrm{kg}^{-1} \cdot \mathrm{fw}\right.$, HPLC-FD) [104] & $\leq 0.3\left(\mathrm{mg} \cdot \mathrm{g}^{-1} \cdot \mathrm{dw}, \mathrm{HPLC}-\mathrm{UV}-\mathrm{DAD}\right)[97,99]$ & - \\
\hline Mv-3-O-(6'-p-coumaroyl)-Glc & 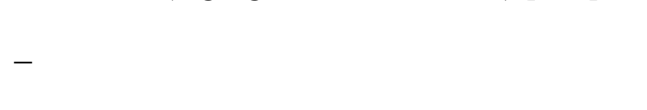 & $\begin{array}{l}\leq 27.10\left(\mathrm{mg} \cdot \mathrm{g}^{-1} \cdot \mathrm{dw}, \text { HPLC-UV-DAD) }[97,99]\right. \\
\leq 0.10\left(\mathrm{mg} \cdot \mathrm{g}^{-1} \cdot \mathrm{dw}, \text { HPLC-DAD) [99] }\right.\end{array}$ & $11.7\left(\mathrm{mg} \cdot \mathrm{g}^{-1} \cdot \mathrm{dw}, \mathrm{HPLC}-\mathrm{DAD}\right)[61]$ \\
\hline Pn-3-O-Glc & $\begin{array}{l}1.90-7.10\left(\mathrm{mg} \cdot \mathrm{g}^{-1} \cdot \mathrm{dw}, \text { HPLC-DAD) [99] }\right. \\
0.30-11.50\left(\mathrm{mg} \cdot \mathrm{g}^{-1} \cdot \mathrm{dw}, \text { HPLC-FD) }[104]\right.\end{array}$ & $\begin{array}{l}0.02\left(\mathrm{mg} \cdot \mathrm{g}^{-1} \cdot \mathrm{dw}, \text { HPLC-UV-DAD) [99] }\right. \\
0.10-1.50\left(\mathrm{mg} \cdot \mathrm{g}^{-1} \cdot \mathrm{dw}, \text { HPLC-UV-DAD) [97] }\right. \\
0.03-3.47\left(\mathrm{mg} \cdot \mathrm{g}^{-1} \cdot \mathrm{dw}, \text { HPLC-DAD) [99] }\right.\end{array}$ & - \\
\hline Pn-3-O-Gal & $0.02-0.10\left(\mathrm{mg} \cdot \mathrm{g}^{-1} \cdot \mathrm{dw}, \mathrm{HPLC}-\mathrm{DAD}\right)[99]$ & - & - \\
\hline Pn-3-O-(6"-O- $p$-coumaryl)-Glc & - & $\leq 1.20\left(\mathrm{mg} \cdot \mathrm{g}^{-1} \cdot \mathrm{dw}\right.$, HPLC-UV-DAD $)[97,99]$ & - \\
\hline
\end{tabular}

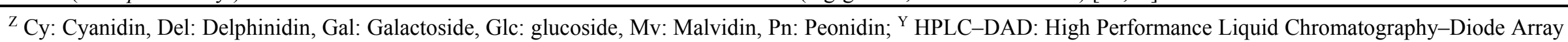
Detector, HPLC-FD: High Performance Liquid Chromatography-Fluorescence Detector, HPLC-UV: High Performance Liquid Chromatography-Ultraviolet Detector; dw, dry weight; fw, fresh weight. 
Table 6. Content in stilbenes (analytical technique employed) in winery residues of red grapes (Vitis vinifera L.).

\begin{tabular}{|c|c|c|c|c|c|}
\hline Compound & Stem & Skin & Seed & Pomace & Leaf \\
\hline trans-Res & $\begin{array}{l}\leq 0.09-124.10\left(\mathrm{mg} \cdot \mathrm{g}^{-1} \cdot \mathrm{dw}\right. \\
\text { HPLC-DAD } \\
\text { Y })[65,81,92,102]\end{array}$ & - & $\leq 0.01\left(\mathrm{mg} \cdot \mathrm{g}^{-1} \cdot \mathrm{dw}, \mathrm{HPLC}-\mathrm{DAD}\right)[33]$ & $\begin{array}{l}\leq 0.06\left(\mathrm{mg} \cdot \mathrm{g}^{-1} \cdot \mathrm{dw}\right. \\
\text { HPLC-DAD) [33] }\end{array}$ & - \\
\hline trans-Res-3-O-Glc ${ }^{\mathrm{Z}}$ & - & - & - & - & $\leq 96.00\left(\mathrm{mg} \cdot \mathrm{kg}^{-1} \cdot \mathrm{fw}, \mathrm{HPLC}-\mathrm{UV}\right)[86]$ \\
\hline cis-Res-3-O-Glc & - & - & - & - & $\leq 129.00\left(\mathrm{mg} \cdot \mathrm{kg}^{-1} \cdot \mathrm{fw}, \mathrm{HPLC}-\mathrm{UV}\right)[86]$ \\
\hline$\varepsilon$-Viniferin & $\begin{array}{l}0.20-49.10\left(\mathrm{mg} \cdot \mathrm{g}^{-1} \cdot \mathrm{dw}\right. \\
\text { HPLC-DAD) }[65,81,102]\end{array}$ & - & - & - & - \\
\hline
\end{tabular}

Z Glc: glucoside, Res: Resveratrol; ${ }^{Y}$ HPLC-DAD: High Performance Liquid Chromatography-Diode Array Detector; HPLC-UV: High Performance Liquid Chromatography-Ultraviolet detector; dw, dry weight; fw, fresh weight.

Table 7. Content in stilbenes (analytical technique employed) in winery residues of white grapes (Vitis vinifera L.).

\begin{tabular}{|c|c|c|c|c|c|}
\hline Compound & Stem & Skin & Seed & Pomace & Leaf \\
\hline trans-Piceid & - & $\leq 6.90\left(\mathrm{mg} \cdot \mathrm{g}^{-1} \cdot \mathrm{dw}, \mathrm{HPLC}-\mathrm{UV}\right)[82]$ & - & - & - \\
\hline trans-Res ${ }^{Z}$ & $\leq 0.02\left(\mathrm{mg} \cdot \mathrm{g}^{-1} \cdot \mathrm{dw}\right.$, HPLC-DAD $\left.{ }^{\mathrm{Y}}\right)[65,81,92]$ & $\leq 1.40\left(\mathrm{mg} \cdot \mathrm{g}^{-1} \cdot \mathrm{dw}\right.$, HPLC-UV) [82] & - & - & - \\
\hline$\varepsilon$-Viniferin & $\begin{array}{l}12.10-50.10\left(\mathrm{mg} \cdot \mathrm{g}^{-1} \cdot \mathrm{dw}, \text { HPLC-DAD) [81] }\right. \\
1.67-4.99\left(\mu \mathrm{g} \cdot \mathrm{g}^{-1} \cdot \mathrm{dw} ; \text { HPLC-DAD) [65] }\right.\end{array}$ & - & - & - & - \\
\hline
\end{tabular}

${ }^{\mathrm{z}}$ Res: Resveratrol; ${ }^{\mathrm{Y}}$ HPLC-DAD: High Performance Liquid Chromatography-Diode array detector; HPLC-UV: High Performance Liquid Chromatography-Ultraviolet detector; dw, dry weight; fw, fresh weight. 
Condensed tannins also represent a significant proportion of the bioactive phytochemicals in vinification residues and available records describe the presence of procyanidin dimers B1, B2, B3, and $\mathrm{B} 4$ and procyanidin trimers $\mathrm{C} 1, \mathrm{C} 2$, and $\mathrm{C} 3$. The analysis of the relative proportions as well as the absolute concentrations of these compounds in the winery by-products suggest critical differences in residues from red and white varieties which are of interest for the evaluation of the further uses of separate materials. Concerning red varieties, the highest content in condensed tannins is represented by the procyanidin dimer B3 (up to $20.50 \mathrm{mg} \cdot \mathrm{g}^{-1} \cdot \mathrm{dw}$ ) $[65,81]$, whereas they are almost absent in grape skins or seeds. However, data from white grape residues shows that although the highest content is found in stems $\left(187.70 \mathrm{mg} \cdot \mathrm{g}^{-1} \cdot \mathrm{dw}\right)$, the concentrations are much higher than those described for red varieties, with procyanidin B1 as the major compound in white grapes. Thus, grape skins and seeds, also showed procyanidin dimer B2 as the most abundant compound with concentrations of total procyanidins up to 8.70 and $6.50 \mathrm{mg} \cdot \mathrm{g}^{-1} \cdot \mathrm{dw}$, respectively (Table 4). Standardized grape seed extracts contain between $74 \%$ and $78 \%$ oligomeric proanthocyanidins, on a dry weight basis [113], which can combine with gallic acid to form gallate esters, and ultimately glycosides [8,114].

Based on these facts, the differences between the main procyanidin in the different grape tissues could seem contradictory. In order to understand these differences it is necessary to take into account that, in the case of procyanidins B3 and B4, certain deviations in the results reported is possible, since these were quantified with the response factor of procyanidin B1 given to the lack of specific commercial standards. This response factor in the HPLC methods varies for available standards of procyanidins B1 and B2 and, therefore, a similar variation would be likely for the response factor of procyanidins B3 and B4 [87].

Condensed tannins represent a quantitatively important value of the pomace composition $(21 \%-52 \%$ of the dry weight matter) even though, until now, the number of publications on the quantification of individual compounds in grape pomace does not allow the comparison with other tissues. Proanthocyanidin oligomers are presumably linked through covalent bonding to cell wall polysaccharide materials, and may explain the high tannin content of the residual pomace [4].

Additional effects of the climate factors should be considered to explain the apparent discrepancy between separate studies. Regarding this matter, analyses of grape procyanidins from plant material obtained in regions with hot summers (typical of Southern Spain and Greece) [106,110], and less extreme summers (Northern Spain and Southern France) $[115,116]$ suggest that climate conditions are also responsible for the differences of the procyanidin patterns.

\subsubsection{Flavones}

Flavones are compounds with a double bond between carbon C2 and C3 that differ from flavonols by the absence of the hydroxyl group in carbon 3. These phytochemicals have been described in non-significant amounts in grapes with the exception of luteolin [68]. Hence, the presence of luteolin has also been reported in winery residues, however, the concentration reported by Çetin et al. (2007) in grape stems is in the range of " $\mu \mathrm{g} \cdot \mathrm{g}^{-1} \cdot \mathrm{dw}$ " with no significant differences between red and white varieties in the hallmark of an evaluation work performed on 10 Turkish cultivars [92]. 


\subsubsection{Anthocyanins}

Anthocyanins are the highly soluble flavonoids directly responsible for color in red grapes and wines, and for which the characteristics are determined by the chemical structure, existing mainly in the stable red coloured form (the flacylium cation form) at low $\mathrm{pH}$ values, degree of hydroxylation, methylation and glycosylation [68,84]. These compounds consist of the aglycone anthocyanidin (cyanidin, delphinidin, and peonidin, among others), an aromatic ring (A) bonded to an heterocyclic ring $(C)$ that contains oxygen, which is also bonded by a carbon-carbon bond to a third aromatic ring (B). This aromatic ring B forms conjugates with sugars and organic acids to generate a multitude of anthocyanins of differing colors. Anthocyanins are relatively unstable and easily oxidized. They are sensitive to many factors, apart from $\mathrm{pH}$, that may affect their stability and colour, such as temperature and UV radiation [117].

Grape skin is the specific part of grape with major content in these bioactive colored flavonoids, this tissue being the most responsible for the transferring of pigments to the wine [82]. Thus, the quantification of anthocyanidins in vinification by-products has been performed on grape skins and pomace (Table 5).

Available data on the distribution of these compounds in grape skin and pomace show that malvidin-3-O-glucoside is the main anthocyanin found with concentrations up to 16.50 and $10.40 \mathrm{mg} \cdot \mathrm{g}^{-1} \cdot \mathrm{dw}$, respectively $[98,99]$. The second most abundant anthocyanin is peonidin-3-O-glucoside, which shows large variations from 0.70 to $11.50 \mathrm{mg} \cdot \mathrm{g}^{-1} \cdot \mathrm{dw}$ in these tissues [104]. Other minor anthocyanins listed in Table 5 have been recorded in much lower concentrations as widely reported [97-99,104]. However, a highly relevant event concerning coloured flavonoids is the biochemical transformation of these compounds during the elaboration of wine and even during wine storage. Hence, during the first steps of fermentation in the winemaking process, anthocyanin-derived pigments, basically pyranoanthocyanins (A- and B-type vitisins and hydroxyphenyl-pyranoanthocyanins derived from $p$-coumaric acid), are formed by the reaction of anthocyanidin-3-glucosinde with compounds of low molecular weight such as 4-vinylphenol, pyruvic acid, and flavonols [105,118]. However, these compounds have been detected in red wines and wine by-products in very minor concentrations, and may be absorbed only by the lees, which display a content of $1.2-2 \mathrm{~g} \cdot \mathrm{kg}^{-1} \cdot \mathrm{dw}$, and not detected in others grape tissues or vinification residues such as grape skin and seeds [119]. Pérez-Serradilla et al. (2011) indicate that antioxidant extracts from wine lees can be a suitable and cheap alternative to those obtained from grape seeds or skins [120].

\subsection{Stilbenes}

Stilbenes are phenolic compounds chemically constituted by two aromatic rings linked by an ethylene bridge [68]. It can be found in wine by-products, mostly in grape skin, but also in stems and seeds but in lower abundance [10,121]. To date, the presence of trans-piceid, trans-resveratrol, trans-resveratrol-3-O-glucoside, $\mathrm{cis}$-resveratrol-3-O-glucoside, and $\varepsilon$-viniferin has been described.

Resveratrol is present in the different organic residues of vinification procedures; the wine processing methods determine its concentration in the final product [122-124]. On this matter, grapes produce stilbenes in response to a number of physiological stressing factors, including ozone and 
UV-C radiation, increasing stilbene levels up to several hundred-folds in grape skins and leaves [125]. Therefore, it may be possible to modify the stilbene contents of grape residues by initial industrial processes used to obtain the must.

The evaluation of the separate organic residues from the winery industry has shown that the content of stilbenes presents a different profile concerning red and white grapes. In this sense, whereas the presence of stilbene derivatives in stems, seeds, pomaces and leaves from red varieties, in white cultivars has been described, they have been detected only in stems and skins [33,65,81,82,86,92,102] (Tables 6 and 7).

The absence of stilbenes in red grape skins as well as their presence only as trans isomers on white grape skins may be influenced by the performance of the extraction procedure (in darkness) since exposure of grapes to UV radiation transforms trans-isomers into cis-isomers [82].

The data available on the concentration of the separate stilbene derivatives indicates that, in red grapes, the highest content corresponded to trans-resveratrol and $\varepsilon$-viniferin in grape stems (up to 124.10 and $49.10 \mathrm{mg} \cdot \mathrm{g}^{-1} \cdot \mathrm{dw}$, respectively) (Table 6), which largely surpasses the concentration recorded in seeds and pomace. Leaves from red varieties do not present these two bioactive stilbenes, showing the presence of the glycosylated forms in the range of $96-129 \mathrm{mg} \cdot \mathrm{kg}^{-1} \cdot \mathrm{fw}$ [86]. On the other hand, grape stems from vinification processes developed on white grapes present lower concentrations of both trans-resveratrol and $\varepsilon$-viniferin (Table 7).

In grapes, trans-resveratrol is distributed mostly in the skin [111], even though the concentration of trans-resveratrol in grape skins and seeds varies considerably within Vitis germplasm [126]. It is worth knowing all resveratrol derivatives in grape by-products in order to support rational valorization of vinification residues for their use in the future. However, only one or two derivatives have been studied along with other phenolic compounds [111,126,127].

\section{Biological Activities and Potential Health Benefits of Winery Wastes Polyphenols}

By-products derived from wine making process contain a high amount of secondary metabolites including phenolic acids, flavanols, proanthocyanidins, flavonols, anthocyanins, and stilbenes [46] (Tables 1-7). To date, there is a great deal of evidence concerning biological activities, such as antioxidant, antimicrobial, anti-inflammatory, anticancer, and cardiovascular protection activities, in support of phenolic compound use in pharmaceutical, food, and cosmetic industries [103,128].

One of the most cited biological activities attributed to the phenolic compounds is based on their antioxidant capacity, preventing the oxidation of LDL lipoproteins, platelet aggregation, and the damage of red blood cells through radical scavenging [129]. Additionally, phenolics are metal chelators, antimutagens/anticarcinogens, antimicrobials, and anti-inflammatory agents $[27,46,130]$.

The radical scavenging activities of phenolic compounds are supported by their reducing power through hydrogen donating and single oxygen quenching. These activities allow these compounds to interact with biological systems, preventing degenerative diseases linked to oxidative stress in separate tissues and organic systems [10,104].

The antioxidant capacity of the (poly)phenolics in vinification residues, to act against oxidative damage [131], support the dietary intake of food products with high content in these natural antioxidants associated with lower prevalence/severity of pathophysiological conditions [132,133]. 
The capacity of the (poly)phenolics in grape derived products to prevent radical oxidation of the polyunsaturated fatty acids of low-density lipoproteins (LDL), which occurs primarily through oxidative modification of the apoprotein toward an atherogenic form, has a direct consequence on the prevention of cardiovascular diseases, appearing to play a major role in this degenerative process [134]. The link between the reduction of oxidized LDL particles and the presence of antioxidant phenolics has been demonstrated in vitro and in vivo and provides evidence for the reduced rates of cardiac diseases in populations consuming diets rich in antioxidant phenolics [135-137].

Anthocyanins contribute more to the antioxidant capacity of fruits (90\%) than flavonols, flavan-3-ols, and phenolic acids (10\%) [138]. On this matter, grape skins have been highlighted for their antioxidant and anti-glycation activities because of their anthocyanins and proanthocyanidins content $[2,33]$. Recent reports showed the antioxidant properties of grape seeds both in vivo and in vitro [21], which has been primarily attributed to flavonoids [139,140], and closely dependent on their chemical structure when present in plant material. Thus, whereas hydroxyl $(-\mathrm{OH})$ groups enhance the activity, their substitution by carboxyl $\left(-\mathrm{OCH}_{3}\right)$ groups diminishes the antioxidant potential [141]. Furthermore, the degree of polymerization of the procyanidins may also determine the antioxidant capacity [142]. The antioxidant capacity of selected grape stem extracts correlated well with the capability to prevent the oxidation of LDL lipoproteins at very low concentrations of (poly)polyphenolics. Thus, grape stem extracts have been suggested for their potential for reducing intracellular ROS levels. However, the poor correlation observed between (poly)phenolics composition and the ability of stem extracts to scavenge intracellular ROS may indicate that total phenolic content could be a poor indicator for the prediction of the antioxidant capacity at the cellular level, which is rationalized by the complexity of mechanisms that occur in living cells [143].

Some preclinical in vivo studies on the capacity of winery residues to prevent LDL oxidation has shown that a $15 \%$ grape pomace in a cholesterol diet $(0.3 \%)$ is able to reduce the rat liver and serum levels of cholesterol by half, while increasing high-density liproprotyeins (HDL) by $26 \%$ [144]. Thus, grape seed extracts have been promoted as a source of (poly)phenols able to reduce the risk of heart disease by inhibiting the oxidation of LDL, improving endothelial function, lowering blood pressure, inhibition of platelet aggregation, reducing inflammation and activating novel proteins that prevent cell senescence [145,146]. Grape seed extract might be also used as a treatment to limit dietary fat absorption and the accumulation of fat in adipose tissue, through its activity on inhibition of the fat-metabolizing enzymes pancreatic lipase and lipoprotein lipase [147]. Additionally, intravenous and oral administration of procyanidins isolated from grape seeds resulted in significant inhibition of thrombus formation in mice models [148]. Similarly, grape seed extracts have demonstrated protection against myocardial ischaemia-reperfusion and myocardial injury in rats as well as reduction in platelet adhesion, aggregation and generation of superoxide anion [149-151].

Concerning oxidative stress in live cells, procyanidins from grape pomace inhibits human endothelial NADPH oxidase, the enzyme responsible for the increased production of reactive oxygen species, inhibiting ROS production at both extra- and intracellular levels [152].

In addition to the radical scavenging capacity, other valuable health promoting activities of (poly)phenols present in grape by-products has been described. Thus, concerning grape pomace and seeds, (poly)phenolic extracts have shown effective antimicrobial capacity; they are efficient against Gram-positive bacteria with medical interest (Staphylococcus aureus, Bacillus cereus, Bacillus subtilis 
and Bacillus coagulans), but more effective against Gram-negative bacteria such as Escherichia coli or Pseudomonas aeruginosa [153]. Similarly, grape pomace extracts have also shown antifungal activity against Botrytis cinerea [154].

Based on the experiments performed on the evaluation of antimicrobial activity of grape seed extracts rich in polymers of flavan-3-ols, the antimicrobial properties of grape tissue extracts has been attributed to the general mechanisms of phenolics. On this matter, Anastasiadi et al. (2009), demonstrated that the high concentrations of flavonoids and their derivatives in grape seeds, as well as the content in flavonoids, stilbenes, and phenolic acids in grape stems, are responsible for the antimicrobial activity of extracts from separate plant materials [155]. Furthermore, data provided by Vaquero et al. (2007), suggested that caffeic acid, quercetin, and quercetin-3-O-rutinoside are responsible for the high inhibitory capacity against Listeria monocytogenes [156]. In the same way, it has been demonstrated that polymeric phenolic fractions produced the highest specific inhibition activity for all Listeria species, but not for other pathological bacteria, such as Bacillus cereus, Escherichia coli, Staphylococcus aureus or Yersinia enterocolitica [157]. More work in this field has reported that the red grape seed extract inhibited the growth of Candida albicans, Escherichia coli, Salmonella typhimurium, and Listeria monocytogenes [158-160]. The study of Paulo et al. (2010), verified the antibacterial activity of grape phenolics attributing the antimicrobial effect to bacteriostatic action by changes in cell morphology and DNA content [161]. Therefore, the cell cycle is affected by grape phenolics. In addition to the direct antimicrobial activity by direct interaction, the cytotoxic effect of extracts of vinification waste residues against bacteria of medical interest in vivo would be due to immunologic induction by such polyphenols [162].

In addition to the mentioned health attributions as antioxidant and antimicrobial agents, phenolic compounds have been also pointed out as interesting bioactives for cardiovascular diseases by means of the inhibition of plasma platelet aggregation and cyclooxygenase activity, the suppression of histamine release and slow-reacting substances of anaphylaxis biosynthesis in vitro, a potent nitric oxide radical scavenging activity, and anti-inflammatory activities [88].

Moreover, grape seeds extracts and their constituents have been proven as inflammatory-preventive with protective effects on chemical-induced ulcerative colitis in rats [163-166]. This study evaluated the protective role of dietary grape seeds extracts on inflammatory bowel disease, attributed to their capacity to modulate gut microflora, decreasing Faecalibacterium prausnitzii within the intestinal lumen and blocking gut inflammatory response, indicating the proanthocyanidins in grape seeds extracts as the key components for the observed beneficial effects.

Additionally, chronic inflammation causes oxidative stress in the affected tissues, and in turn, elevated oxidative stress enhances the inflammatory response by activating redox-sensitive nuclear transcription factors [167]. Dietary antioxidants may provide a cost-effective strategy to promote health through minimizing the severity of oxidative stress and chronic inflammation. On this matter, animal and human clinical trials further demonstrated an improvement of systemic inflammation after antioxidant supplementation $[168,169]$. Since grape pomace contains significant amounts of antioxidants anthocyanins, catechin, epicatechin, quercetin and a few phenolic acids, this material has been suggested as beneficial for the prevention of oxidative stress and inflammatory conditions. This has been further supported at preclinical level by dietary supplementation with $250 \mathrm{mg}$ grape pomace $/ \mathrm{kg}$ per day for 12 weeks, showing significant anti-inflammatory effects in obese mice [170]. 
It has also been shown that grape seed extracts can provide a modulatory effect on age-related oxidative DNA damage and lipid peroxidation concerning the central nervous system in rats $[171,172]$. The health effect of dietary supplementation with this vinification residue was demonstrated by improved memory performance, reduced reactive oxygen species production, decreased protein carbonyl level, increased thiol level, and reduced hypoxic ischaemic brain injury in central nervous systems. In this regard, additional studies have shown that resveratrol could exert neuroprotection against ischaemia, seizure, and neurodegenerative diseases [173].

\section{Current Extractive Techniques for Bioactive Phytochemicals of Industrial By-Products: Limitations and Possibilities to Improve}

The suitability of the separate extractive techniques constitutes a critical issue to gain rational valorization of winemaking by-products. Thus, the qualitative and quantitative composition of the (poly)phenolics extracts from winery by-products depend not only on the source of the plant material concerning the cultivar, the agro-climatic conditions of the production area, and the tissue considered (leaves, stems, seeds, peels, etc.), but also on the physico-chemical conditions of the industrial process, as well as the combination of solvents and the extraction procedures employed. Additional factors such as the quality of the plant material, the storage conditions and its pretreatment are all decisive to define the biochemical profile of the phenolic extracts and, therefore, for the potential biological activity. Hence, all these parameters should be taken into account, in order to produce high quality extracts with valuable biological activities, suitable to be used by food, cosmetic and/or pharmaceutical industries.

Given the final purpose of the valorization of vinification residues based on the functional properties of the bioactive compounds isolated, it is necessary to purify the extract obtained, removing all the inert and undesirable components that could interfere with the final biological activity (radical scavenging, antimicrobial activity, and so on) of the extracts. This procedure will allow reduction of its odour, taste, and colour as much as possible to avoid interference with the technological processes [49]. This approach supports that organic residues (stems, seeds, peels and/or pomace) derived from the vinification process are studied to gain the most effective extraction procedures focused on minimizing the extraction time, reducing organic solvent consumption, and maintaining the recovery of compounds of interest.

To date, there exists a panel of extraction techniques to isolate phenolic compounds from the wine-making residues. The most widespread technique used to extract the (poly)phenolics from winery residues is the solvent solid/liquid extraction (SLE), even though additional procedures, namely supercritical fluids extraction (SFE), ultrasound assisted extractions (UAE), microwave assisted extractions (MAE), and high pressure and temperature extraction (HPTE) have been also assayed providing successful outcomes on phenolic extraction [174].

Regarding SLE methods, a myriad of factors have been described as involved in their efficiency, such as the type of solvent, relative solvents proportion, solvent/sample ratio, particle size, type of phenols under study, temperature, and extraction time, among others, and, therefore, there is not a single optimal extractive condition or procedure for all the types of residues derived from this agro-industrial activity. 
Thus, the comparison of the suitability of the distinct methods assayed so far shows that hydroalcoholic solutions provides quite high yield of total extracts, although they are not highly selective for the distinct classes of phenolics present in the residues [52,175-177]. Another solvent widely employed in the extraction of winery waste phenolics is methanol $(\mathrm{MeOH})$, which obtains high concentrations of these target analytes. However, the toxicity associated to this solvent restricts its use to analytical procedures. In this regard, ethanol $(\mathrm{EtOH})$ has been studied as a more environmentally friendly solvent, recognized as safe according to the European Food Safety Authority (EFSA) and FAO/WHO Expert Committee on Food Additives [178,179]. Concerning the comparison between distinct solvents, recently, the effect of solvent systems $(\mathrm{MeOH}$ and $\mathrm{EtOH})$ on the efficiency of the extraction of total phenolic compounds, total flavonoids, and individual phenolics, as well as on the antioxidant capacity of the extracts obtained from grape pomace, and its constituents (peduncle, skin and seed) has been further investigated [175]. This analysis described the close relation between the solvent used and the specific residue considered in order to get the highest yield of different compounds. This work showed $\mathrm{MeOH}$ as the solvent with the highest extracting capacity (Table 8). On this matter, Lapornik et al. [177] reported that ethanol/water and methanol/water extracts (both at ratio $7: 3, v / v)$ of grape pomace had seven times higher values than water extracts, with $12 \mathrm{~h}$ generally being the appropriate time of extraction.

The wide variety of physical properties involved with the distinct organic matter resulting from vinification processes has prompted optimization of extraction procedures using alternative solvents. Hence, when considering winery derived materials with high content in lipids, the use of ethyl acetate (EtAc) demonstrated the highest efficiency because of its low polarity $[101,160,180]$. Furthermore, the low boiling point of EtAc facilitates its removal and reuse, and any possible residue is scarcely toxic at the remaining concentration [101].

Other critical factors affecting the extraction of phenolic compounds is the extraction time. In this regard, Bonilla et al. (1999) studied the extraction efficiency at four different times 5, 10, 20 and 30, in crushed and uncrushed grape pomace with EtAc and water solvents [101]. As a result, crushing the grape pomace favored the extraction of ratio for both solvents. The anthocyanins are not EtAc-soluble, allowing the separation of colorants (in the aqueous phase) from other phenols (organic phase). Concerning extraction time, $10 \mathrm{~min}$ for the organic extracts and $20 \mathrm{~min}$ for the aqueous extracts of crushed pomace were adopted as the optimum settings. The organic phase was found to contain high amounts of phenols, which have antioxidant lipid activity reasonably lower than synthetic additives. However, the dried phenols extract could be used at higher levels, thereby increasing their antioxidant effectiveness as well as biological activity with beneficial results for human health. Bucic-Kojic et al. optimized the yield and kinetics of the phenolics extraction for grape seeds using Peleg's mathematical model, definitely influenced by temperature, particle size and solid-liquid ratio, with extraction time not being an important parameter after 40 min of treatment [181]. 
Table 8. Polyphenol extraction techniques and solvents for industrial application of wine-industry by-products.

\begin{tabular}{|c|c|c|c|c|}
\hline Extractive Procedure & Vinification Residue & Effective Solvent & Target Compound/Assay & Ref. \\
\hline \multirow{17}{*}{ Solvent extraction } & \multirow{5}{*}{ Grape pomace } & EtOH/Water $(6: 4, v / v)$ & Total phenolic compounds & [175] \\
\hline & & EtOH/Water $(7: 3, v / v)$ & Total flavonoid compounds & [175] \\
\hline & & Ethyl acetate/Water $(1: 1, v / v)$ & Anthocyanins (aqueous phase) and phenolics (organic phase) & [100] \\
\hline & & Ethyl acetate/water $(9: 1, v / v)$ & Total phenolic compounds & [176] \\
\hline & & $\mathrm{MeOH} /$ water $(7: 3, v / v)$ and EtOH/water $(7: 3, v / v)$ & Total phenolic compounds & [177] \\
\hline & \multirow{2}{*}{ Grape seeds } & $\mathrm{MeOH} /$ Water/Acetone $(3: 3.5: 3.5, v / v / v)$ & Total phenolic compounds, total flavonoids, quercetin-3-rutinoside, myricetin & [175] \\
\hline & & $\mathrm{MeOH} /$ Water $(7: 3, v / v)$ & Catechin, epicatechin & [175] \\
\hline & Grape skins & $\mathrm{MeOH} /$ Water/Acetone $(3: 3.5: 3.5, v / v / v)$ & Total phenolic compounds, total flavonoids, myricetin & [175] \\
\hline & \multirow{2}{*}{ Grape skins } & $\mathrm{MeOH} /$ Ethyl acetate $(1: 1, v / v)$ & Resveratrol & [121] \\
\hline & & $\mathrm{MeOH} /$ Water $(7: 3, v / v)$ & Quercetin and quercetin-3-rutinoside & [175] \\
\hline & \multirow{3}{*}{ Grape peduncules } & $\mathrm{MeOH} /$ Water/Acetone $(3: 3.5: 3.5, v / v / v)$ & Quercetin & [175] \\
\hline & & $\mathrm{MeOH} /$ Water $(7: 3, v / v)$ & Quercetin-3-rutinoside, myricetin, catechin & [175] \\
\hline & & $\mathrm{MeOH} /$ Water $(6: 4, v / v)$ & Total phenolic compounds, total flavonoids & [175] \\
\hline & \multirow{3}{*}{ Grape stems } & EtOH/Water $(6: 6, v / v)$ & Flavones & {$[52]$} \\
\hline & & EtOH/Water $(6: 4, v / v)$ & Flavonols & {$[52]$} \\
\hline & & EtOH/Water $(4.5: 5.5, v / v)$ & Proanthocyanidins & {$[52]$} \\
\hline & Grape pomace/stems & EtOH/Water $(9: 1, v / v)$ & Total phenolic compounds & [176] \\
\hline \multirow{2}{*}{ Solvent extraction and supercritical fluid extraction } & Grape pomace & Ethyl acetate and SFE $\mathrm{CO}_{2}$ & Total phenolic compounds & {$[49]$} \\
\hline & Grape seeds & $\mathrm{EtOH}$ and $\mathrm{SFE} \mathrm{CO}_{2}$ & Total phenolic compounds & [182] \\
\hline \multirow{2}{*}{ Microwave assisted exraction } & Grape seeds & $70 \mathrm{~W}, \mathrm{MeOH}$ & Quercetin, Catechin & [158] \\
\hline & Grape skins & $500 \mathrm{~W}, \mathrm{MeOH}:$ water $(6: 4, v / v)$ & Anthocyanins & [183] \\
\hline \multirow{2}{*}{ Ultrasound assisted extraction } & \multirow{2}{*}{ Grape skins } & $35 \mathrm{KHz}, \mathrm{MeOH} / \mathrm{HCl}(99: 1, v / v)$ & Anthocyanins, flavan-3-ols, and flavonols & [167] \\
\hline & & $35 \mathrm{KHz}, \mathrm{EtOH} /$ Water $(1: 1, v / v)$ & Anthocyanins, total phenolic compounds & {$[46]$} \\
\hline High pressure and temperature extraction & Grape seeds & Reactor $\left(350^{\circ} \mathrm{C} / 200\right.$ bar $)$ & Gallic acid, hydroxytyrosol, vanillic acid, syringic acid, and trans-resveratrol & [184] \\
\hline Aqueous $\beta$-cyclodextrins & Grape pomace & $2.5 \%(w / v)$ aqueous $\beta$-CD solutions & Individuals flavonols, flavan-3-ols, stilbenes, and ortho-diohenols & [109] \\
\hline
\end{tabular}


The effectiveness of the solvent not only depends on the physical properties of the residue considered, but also on the target phenolic to be extracted. Thus, the extraction of bioactive phenolics present in grape seeds using EtAc as solvent, showed that this solvent largely recover flavonols, whereas methanol has been reported to be the best solvent for extracting flavan-3-ols (catechin, epicatechin, and epillocatechin). The use of ethyl acetate or acetone with addition of water resulted in a significantly increased yield of extracted proanthocyanidins. This is a consequence of a low permeability of the sample tissues to only EtAc or $\mathrm{MeOH}$, which is a non-polar aprotic solvent [184].

Even though the most evaluated winery residue is grape pomace, further analyses on other organic material from this industrial activity have pointed out the relevance of less studied materials as interesting sources of bioactive compounds. Thus, Spigno et al. showed grape stems as valuable source of antioxidant phytochemicals, showing a high purity of phenolic extracts from grape stems by using EtAc [185]. The use of different combinations of $\mathrm{MeOH}$, EtOH and acetone $\left(\mathrm{C}_{3} \mathrm{H}_{6} \mathrm{O}\right)$ with water, generally, yields a significant co-extraction of concomitant substances, which decreases the final content in target bioactives in the extract, and complicates the purification.

Again, time and temperature highly influenced the success of the extraction procedure. When considering these factors, the best results were obtained by increasing temperature up to $60{ }^{\circ} \mathrm{C}$, because at this temperature the solubility of solutes and diffusion coefficient increases without decreasing the phenolics' stability. Nevertheless, temperature cannot be considered as an isolated factor, being closely linked to the extraction time $(8 \mathrm{~h})$ as a factor that should be controlled to upgrade processes from the bench to the industry [185]. In this sense, a number of works on the optimization of the panel of factors influencing the efficiency of the extraction process of (poly)phenolic compounds from winery residues based on the minimization of the experimental conditions as a time-saving approach, provided by the response surface methodology has recently been reported $[52,186]$.

Furthermore, supercritical fluid extraction (SFE) has been pointed out as an environmentally friendly alternative to extract polyphenols since it avoids the use of large amounts of toxic solvents. In this regard, supercritical $\mathrm{CO}_{2}$ working at 150 bar and $30-40{ }^{\circ} \mathrm{C}$ has been employed for obtaining primary extract using ethyl acetate as extraction solvent, in order to improve its properties (high antioxidant activity, lighter colour, and no odour) without causing any thermal or chemical degradation, as $\mathrm{CO}_{2}$ is an inert, non-toxic, and volatile solvent. This extraction technique obtains high added-value products under moderate conditions and equipment capacity [185].

Concerning ultrasound and microwave assisted extractions (UAE and MAE), they are recognized as outstanding energy sources to promote extraction, cutting down extraction times and increasing yields as well as the quality of the extract in terms of phenolic composition and biological activity. The UAE and MAE are chiefly exploited at laboratory scale and both have already been addressed toward industrial applications [15]. When comparing these extraction methods with conventional ones, the UAE appears to be much faster than traditional procedures, such as SLE, because UAE provides a higher contact-surface area between solid and liquid phase as a consequence of the fine reduction of particle size. Moreover, acoustic cavitation of power US (frequencies range 18-40 kHz), causes cell wall disruption, enhancing mass transfer and facilitating solvent access to the cell content [187]. On the other hand, the MAE employs the microwave energy to heat polar solvents in contact with organic residues from the winery industry (grape seeds, grape skins, and so on), reducing both extraction time and solvent consumption. The MAE are performed at high temperatures $\left(110-150{ }^{\circ} \mathrm{C}\right)$, 
which constitutes a critical feature to handle antioxidants concerning to degradative conditions [188]. The comparison of the data available in the bibliography on the (poly)phenolic extraction from grape pomace by SLE, MAE, UAE, and high pressure and temperature extraction (HPTE) methods shows higher yields of total phenolics when using methanol rather than ethanol in SLE. Furthermore, when using methanol, HPTE yielded higher extraction of phenolics than UAE and SLE, MAE being the second most effective technique. These reports suggested the critical role of the extraction time, since the increase of the contact time from 15 to 30 min leads to total phenolic content increase, whereas its extension up to $90 \mathrm{~min}$ resulted in a decrease of their extraction. Delgado-Torre, et al. also compared UAE, MAE, and superheated liquid extraction (SHLE) in different samples of grape stems, and showed that SHLE was the best extractive option for total phenolics [16].

Recently, aqueous $\beta$-cyclodextrins (CD) has also been studied as a tool for recovering phenolics from wine-industry by-products directed to be used as ingredients in the development of innovative added-value foods and nutraceuticals. Due to its specific structure, the CDs are able to form host-guest inclusion complexes with a wide range of phenolic compounds, increasing their water solubility, stability, bioactivity, and bioavailability. The effective extraction procedure was achieved with $2.5 \%$ $(w / v)$ aqueous $\beta$-CDs solutions at $60{ }^{\circ} \mathrm{C}$ for $12-24 \mathrm{~h}$ (Table 8) [183].

Given the constraints of the industrial production as well as the seasonality of the winery by-products availability, extracts from this agro-economic activity required preservation for further use in applications and valorization processes. Thus, once extracted, bioactive compounds could be stored as dried extracts. This physical state displays advantages over liquid forms such as lower storage costs, stability of active phytochemicals, and their versatility to be integrated in further processes in the required concentration. On this matter, one of the drying techniques most widely used by the food industry is the spray-drying, which was optimized by Pérez-Serradilla et al. 2011, allowing antioxidant wine lees extracts to be obtained in powder form with similar physicochemical characteristics than those of the grape seed extracts [61].

All data recorded on the beneficial properties of extracts from winery residues are receiving attention not only from the scientific community but also from sectorial industries, involved in the valorization of resources of bioactive compounds, in order to gain further insight into the exploitation of these multipurpose materials concerning cosmetic, pharmaceutical, and dietary supplement uses. This trend has allowed the development of patents, such as for wine waste extracts for reducing plasma triglycerides, platelet aggregation, and oxidative capacity [189].

Additional uses of these under-profit materials are supported by the beverage industries. Thus, dried grape skins may be interesting ingredients for teas and other health promoting beverages (for example, isotonic drinks) since grapes are very aromatic fruits with wide flavour properties [190,191].

\section{Conclusions}

Consumption of grapes and grape-based foods is recognized as a major contributor to the beneficial effects of the "Mediterranean Diet". Currently, grapes and their derivatives have attracted scientific interest to confirm their applications in the development of innovative added-value products. A relevant proportion of grapes (up to $15 \%$ of yields) is directed to the winery industry, which is linked to the production of high amounts of undesirable by-products, which include seeds, peels, and 
grape stems. These have been also evaluated for their content in bioactive compounds revealing that several factors, such as cultivar, agro-climatic conditions and degree of ripening, among others, are responsible for the wide variations in their phytochemical profiles. Accurate data on the biological functions of these compounds available nowadays have allowed their identification as responsible agents for multiple benefits involved in the prevention of degenerative processes through their integration into functional foods, nutraceuticals, and cosmetics. Hence, the most relevant activities attributed to bioactive phytochemicals from winery by-products are antioxidant, antimicrobial, anti-inflammatory, and anticancer $[41,81,157,172]$. Even though vinification residues are considered in general, a good source of bioactive compounds, the total amount of phenolics, as well as the specific profile concerning compound identity and their relative proportion, is strongly dependent on the type of residue considered. In this regard, the identification of the specific (poly)phenols of the separate winery wastes has prompted the development of more extensive panels of valorization options for these residues. Thus, recycling of winery co-products or side streams constitutes an opportunity for providing valuable materials to pharmaceutical, cosmetic, nutraceutical, and food industries, contributing to reduced costs and environmental impact linked to the disposal of these by-products in the production areas.

\section{Future Prospects}

Over the last decades a large knowledge base on the bioactivity, bioavailabily and toxicology of phytochemicals has emerged. This has allowed the relevance of bioactive compounds as health promoters in the hallmark of healthy diets and lifestyles to be enhanced. To gain knowledge on the potential of bioactives to reduce the incidence and/or severity of degenerative diseases, their interaction within their food matrices related to the dietary administration as well as the specific bioavailability in pharmacological or food formulas needs studies under in vitro and in vivo conditions. Winery by-products are good sources of phytochemicals and natural antioxidants, and partially exploited for their health promoting properties mainly in the cosmetic industry. However, even though some applications of these agro-industrial by-products have been found to date, their valorization needs additional commitment concerning the development of novel extraction, isolation, purification, and recovery procedures to obtain higher amounts of healthy bioactive phytochemicals and to deliver standardized potency in different fields. Efforts should also be focused on the isolation and structural elucidation of novel grape phenolic compounds from the less characterized materials derived from vinification processes (grape stems), using chromatographic and spectroscopic tools. In this sense, adequate procedures concerning the target product and time-saving technologies to allow the integration of the obtained results for sectorial industries, will provide successful achievements for phytochemical recovery and innovative products, which could contribute to the reduction of environmental pressure in winery areas from winery by-products, and promote the application of isolated bioactives in feed, food, pharmaceutical, and cosmetic industries.

\section{Acknowledgments}

This work was supported by national funds from FCT-Portuguese Foundation for Science and Technology, under the projects PEst-OE/AGR/UI4033/2014 and Project INNOFOOD-INNovation in the FOOD sector through the valorization of food and agro-food by-products-NORTE-07-0124- 
FEDER-0000029, financed by the North Portugal Regional Operational Programme (ON.2-O Novo Norte) under the National Strategic Reference Framework (QREN), through the European Regional Development Fund (FEDER). The authors also express their gratitude to the Spanish Ministry of Economy and Competitiveness for the funding through the CICYT project AGL2011-23690, and the CYTED Program (Ref. 112RT0460) CORNUCOPIA Thematic Network [192].

\section{Abbreviations}

BHT, Buthylated hydroxytoluene; BHA, Buthylated hydroxyanisole; Cy, Cyanidin; Del, Delphinidin; dw, dry weight; fw, fresh weight; GAE, Galic acid equivalents; Gal, Galactoside; Glc, Glucoside; Gluc, Glucuronide; HDL, High-density lipoprotein; HPLC-DAD, High Performance Liquid Chromatography-Diode Array Detector; HPLC-FD, High Performance Liquid ChromatographyFluorescence Detector; HPLC-UV, High Performance Liquid Chromatography-Ultraviolet Detector; HPTE, High pressure and temperature extraction; Isrhm, Isorhamntin; LDL, Low-density lipoprotein; K, Kaempferol; MAE, Microwaves assisted extractions; Mv, Malvidin; Myr, Myricetin; Pn, Peonidin; Q, Quercetin; Res, Resveratrol; Rha, Rhamnoside; Rut, Rutinoside; ROS, Reactive Oxygen Species; SFE, Supercritical fluids extraction; SLE, Solvent extraction; TBHQ, tert-buthylhydroxyquinone; UAE, Ultrasound assisted extractions; Xyl, Xyloside.

\section{Conflicts of Interest}

The authors declare no conflict of interest.

\section{References}

1. FAOSTAT, Food and Agriculture Organization of the United Nations. Available online: http:/faostat3.fao.org/faostat-gateway/go/to/download/Q/QC/E (accessed on 2 March 2014).

2. Poudel, P.R.; Tamura, H.; Kataoka, I.; Mochioka, R. Phenolic compounds and antioxidant activities of skins and seeds of five wild grapes and two hybrids native to Japan. J. Food Comp. Anal. 2008, 21, 622-625.

3. Musee, N.; Lorenzen, L.; Aldrich, C. Cellar waste minimization in the wine industry: A systems approach. J. Clean. Prod. 2007, 15, 417-431.

4. Rondeau, P.; Gambier, F.; Jolibert, F.; Brosse, N. Compositions and chemical variability of grape pomaces from French vineyard. Ind. Crops Prod. 2013, 43, 251-254.

5. Brito, A.G.; Peixoto, J.; Oliveria, J.M.; Oliveria, J.A.; Costa, C.; Nogueira, R.; Rodrigues, A. Brewery and winery wastewater treatment: Some focal points of design and operation. In Utilization of By-Products and Treatment of Waste in the Food Industry. Oreopoulou, V., Russ, W., Eds.; Springer Science + Business Media Llc.: New York, NY, USA, 2007; pp. 109-131.

6. Saura-Calixto, F. Antioxidant dietary fiber product: A new concept and a potential food ingredient. J. Agric. Food Chem. 1998, 46, 4303-4306.

7. Shrikhande, A.J. Wine by-products with health benefits. Food Res. Int. 2000, 33, 469-474.

8. Negro, C.; Tommasi, L.; Miceli, A. Phenolic compounds and antioxidant activity from red grape marc extracts. Bioresour. Technol. 2003, 87, 41-44. 
9. Corrales, M.; Fernandez, A.; Pinto, M.G.V.; Butz, P.; Franz, C.M.A.P.; Schuele, E.; Tauscher, B. Characterization of phenolic content, in vitro biological activity, and pesticide loads of extracts from white grape skins from organic and conventional cultivars. Food Chem. Toxicol. 2010, 48, 3471-3476.

10. Kataliníc, V.; Možina, S.S.; Skroza, D.; Generalić, I.; Abramovič, H.; Miloš, M.; Ljubenkov, I.; Piskernik, S.; Terpinc, P.; Boban, M. Polyphenolic profile, antioxidant properties and antimicrobial activity of grape skin extracts of 14 Vitis vinifera varieties grown in Dalmatia (Croatia). Food Chem. 2010, 119, 715-723.

11. Soleas, G.J.; Diamandis, E.P.; Goldberg, D.M. Wine as a biological fluid: history, production, and role in disease prevention. J. Clin. Lab. Anal. 1997, 11, 287-313.

12. Singleton, V.L.; Trousdale, E. White wine phenolics-Varietal and processing differences as shown by HPLC. Am. J. Enol. Vitic. 1983, 34, 27-34.

13. Ju, Z.Y.; Howard, L.R. Effects of solvent a temperature on pressurized liquid extraction of anthocyanins and total phenolics from dried red grape skin. J. Agric. Food Chem. 2003, 51, 5207-5213.

14. Ju, Z.Y.; Howard, L.R. Subcritical water and sulfured water extraction of anthocyanins and other phenolics from dried red grape skin. J. Food Sci. 2005, 70, 270-276.

15. Casazza, A.A.; Aliakbarian, B.; Mantegna, S.; Cravotto, G.; Perego, P. Extraction of phenolics from Vitis vinifera wastes using non-conventional techniques. J. Food Eng. 2010, 100, 50-55.

16. Delgado-Torre, M.P.; Ferreiro-Vera, C.; Priego-Capote, F.; Pérez-Juan, P.M.; Luque de Castro, M.D. Comparison of accelerated methods for the extraction of phenolic compounds from different vine-shoot cultivars. J. Agric. Food Chem. 2012, 60, 3051-3060.

17. Oliveira, D.A.; Salvador, A.A.A.S.; Smânia, E.F.A.; Maraschin, M.; Ferreira, S.R.S. Antimicrobial activity and composition profile of grape (Vitis vinifera) pomace extracts obtained by supercritical fluids. J. Biotechnol. 2013, 164, 423-432.

18. Chouchouli, V.; Kalogeropoulos, N.; Konteles, S.J.; Karvela, E.; Makris, D.P.; Karathanos, V.T. Fortification of yoghurts with grape (Vitis vinifera) seed extracts. Food Sci. Technol. 2013, 53, 522-529.

19. Arvanitoyannis, I.S.; Ladas, D.; Mavromatis, A. Potential uses and applications of treated wine waste. Int. J. Food Sci. Technol. 2006, 41, 475-487.

20. Harsha, P.S.C.S.; Gardana, C.; Simonetti, P.; Spigno, G.; Lavelli, V. Characterization of phenolics, in vitro reducing capacity and anti-glycation activity of red grape skins recovered from winemaking by-products. Bioresour. Technol. 2013, 140, 263-268.

21. Yilmaz, Y.; Toledo, R.T. Major flavonoids in grape seeds and skins: Antioxidant capacity of catechin, epicatechin, and gallic acid. J. Agric. Food Chem. 2004, 52, 255-260.

22. González-Centeno, M.R.; Knoerzer, K.; Sabarez, H.; Simal, S.; Rosselló, C.; Femenia, A. Effect of acoustic frequency and power density on the aqueous ultrasonic-assisted extraction of grape pomace (Vitis vinifera L.) - A response surface approach. Ultrason. Sonochem. 2014, 21, 2176-2184.

23. Makris, D.P.; Boskou, G.; Andrikopoulos, N.K. Polyphenolic content and in vitro antioxidant characteristics of wine industry and other Agri-food solid waste extracts. J. Food Comp. Anal. 2007, 20, 125-132. 
24. Torres, J.L.; Varela, B.; García, M.T.; Carilla, J.; Matito, C.; Centelles, J.J.; Cascante, M.; Sort, X.; Bobet, R. Valorization of grape (Vitis vinifera) by-products. Antioxidant and biological properties of polyphenolic fractions differing in procyanidin composition and flavonol content. J. Agric. Food Chem. 2002, 50, 7548-7555.

25. Laufenberg, G.; Kunz, B.; Nystroem, M. Transformation of vegetable waste into value added products: (A) the upgrading concept; (B) practical implementations. Bioresour. Technol. 2003, 87, 167-198.

26. Panouillé, M.; Ralet, M.-C.; Bonnin, E.; Thibault, J.-F. Recovery and reuse of trimmings and pulps from fruit and vegetable processing. In Handbook of Waste Management and Co-Product Recovery in Food Processing; Woodhead Publishing: Abington Hall, UK, 2007; pp. 417-447.

27. Boussetta, N.; Lanoisellé, J.L.; Bedel-Cloutour, C.; Vorobiev, E. Extraction of soluble matter from grape pomace by high voltage electrical discharges for polyphenol recovery: Effect of sulphur dioxide and thermal treatments. J. Food. Eng. 2009, 95, 192-198.

28. González-Centeno, M.R.; Rosselló, C.; Simal, S.; Garau, M.C.; López, F.; Femenia, A. Physico-chemical properties of cell wall materials obtained from tem grape varieties and their byproducts: Grape pomace and stems. LWT-Food Sci. Technol. 2010, 43, 1580-1586.

29. Brahim, M.; Gambier, F.; Brosse, N. Optimization of polyphenols extraction from grape residues in water medium. Ind. Crop Prod. 2014, 52, 18-22.

30. Deng, Q.; Penner, M.H.; Zhao, Y. Chemical composition of dietary fiber and polyphenols of five different varieties of wine grape pomace skins. Food Res. Int. 2011, 44, 2712-2720.

31. Sánchez, M.; Franco, D.; Sineiro, J.; Magariños, B.; Nuñez, M.J. Antioxidant power, bacteriostatic activity, and characterization of white grape pomace extracts by HPLC-ESI-MS. Eur. Food Res. Technol. 2009, 230, 291-301.

32. Rockenbach, I.I.; Rodrigues, E.; Gonzaga, L.V.; Caliari, V.; Genovese, M.I.; Gonçalves, A.E.S.S.; Fett, R. Phenolic compounds content and antioxidant activity in pomace from selected red grapes (Vitis vinifera L. and Vitis labrusca L.) widely produced in Brazil. Food Chem. 2011, 127, 174-179.

33. Rockenbach, I.I.; Gonzaga, L.V.; Rizelio, V.M.; Gonçalves, A.E.S.S.; Genovese, M.I.; Fett, R. Phenolic compounds and antioxidant activity of seed and skin extracts of red grape (Vitis vinifera and Vitis labrusca) pomace from Brazilian winemaking. Food Res. Intern. 2011, 44, 897-901.

34. Ghafoor, K.; Choi, Y.H.; Jeon, J.Y.; Jo, I.H. Optimization of ultrasound-assisted extraction of phenolic compounds, antioxidants, and anthocyanins from grape (Vitis vinifera) seeds. J. Agric. Food Chem. 2009, 57, 4988-4994.

35. Nawaz, H.; Shi, J.; Mittal, G.S.; Kakuda, Y. Extraction of polyphenols from grape seeds and concentration by ultrafiltration. Sep. Purif. Technol. 2006, 48, 176-181.

36. Stamatina, K.; Garcia-Viguera, C.; Bridle, P.; Bakker, J. Survey of solvents for the extraction of grape seed phenolics. Phyto. Anal. 1995, 6, 265-267.

37. Campos, L.M.A.S.; Leimann, F.V.; Pedrosa, R.C.; Ferreira, S.R.S. Free radical scavenging of grape pomace extracts from Cabernet Sauvingnon (Vitis vinifera). Bioresour. Technol. 2008, 99, 8413-8413.

38. González-Manzano, S.; Rivas-Gonzalo, J.C.; Santos-Buelga, C. Extraction offlavan-3-ols from grape seed and skin into wine using simulated maceration. Anal. Chim. Acta 2004, 513, 283-289. 
39. Ariga, T. The antioxidative function, preventive action on disease and utilization of proanthocyanidins. Biofactors 2004, 21, 197-201.

40. Bucić-Kojić, A.; Sovová, H.; Planinić, M.; Tomas, S. Temperature-dependent kinetics of grape seed phenolic compounds extraction: Experiment and model. Food Chem. 2013, 136, 1136-1140.

41. Furiga, A.; Lonvaud-Funel, A.; Badet, C. In vitro study of antioxidant capacity and antibacterial activity on oral anaerobes of a grape seed extract. Food Chem. 2009, 113, 1037-1040.

42. Escarpa A.; González, M.C. High-performance liquid chromatography with diode-array detection for the determination of phenolic compounds in peel and pulp from different apple varieties. J. Chromatgr. A 1998, 823, 31-37.

43. Pinelo, M.; Arnous, A.; Meyer, A.S. Upgrading of grape skins: Significance of plant cell-wall structural components and extraction techniques for phenol release. Trends Food Sci. Technol. 2006, 17, 579-590.

44. Llobera, A.; Canellas, J. Dietary fiiet content and antioxidant activity of Manto Negro red grape (Vitis vinifera): Pomace and stem. Food Chem. 2007, 101, 659-666.

45. Souquet, J.M.; Labarbe, B.; Guernevé, C.L.; Cheynier, V.; Moutounet, M. Phenolic composition of grape stems. J. Agric. Food Chem. 2000, 48, 1076-1080.

46. Corrales, M.; Toepfl, S.; Butz, P.; Knorr, D.; Tauscher, B. Extraction of anthocyanins from grape by-products assisted by ultrasonics, high hydrostatic pressure or pulsed electric fields: A comparison. Innov. Food Sci. Emerg. Technol. 2008, 9, 85-91.

47. Figuerola, F.; Hurtado, M.L.; Estévez, A.M.; Chiffelle, I.; Asenjo, F. Fibre concentrates from apple pomace and citrus peel as potential fibre sources for food enrichment. Food Chem. 2005, 91, 395-401.

48. Llobera, A.; Canellas, J. Antioxidant activity and dietary fiiet of Prensal Blanc white grape (Vitis vinifera) by-products. Int. J. Food Sci. Technol. 2008, 43, 1953-1959.

49. Louli, V.; Ragoussis, N.; Magoulas, K. Recovery of phenolic antioxidants from wine industry by-products. Bioresour. Technol. 2004, 92, 201-208.

50. Valiente, C.; Arrigoni, C.; Esteban, R.M.; Amado, R. Grape pomace as a potential food fiber. J. Food Sci. 1995, 60, 818-820.

51. Cao, X.; Ito, Y. Supercritical fluid extraction of grape seed oil and subsequentseparation of free fatty acids by high-speed counter-current chromatography. J. Chromatogr. A 2003, 1021, 117-124.

52. Karvela, E.; Makris, D.P.; Kalogeropoulos, N.; Karathanos, V.T. Deployment of response surface methodology to optimise recovery of grape (Vitis vinifera) stem polyphenols. Talanta 2009, 79, 1311-1321.

53. Makris, D.P.; Boskou, G.; Chiou, A.; Andrikopoulos, N.K. An investigation on factors affecting recovery of antioxidant phenolics and anthocyanins from red grape (Vitis vinifera L.) pomace employing water/ethanol-based solutions. Am. J. Food Technol. 2008, 3, 164-173.

54. Bombardelli, E.; Morrazzoni, P. Vitis vinifera L. Fitoterapia 1995, 66, 291-317.

55. Felício, J.D.; Rossi, M.H.; Park, H.R.; Gonçalez, E.; Braggio, M.M.; David, J.M.; Cordeiro, I. Biflavonoids from Ouratea multiflora. Fitoterapia 2001, 72, 453-455.

56. Hebash, K.A.H.; Fadel, H.M.; Soliman, M.M.A. Volatile components of grape leaves. J. Isl. Acad. Sci. 1991, 4, 26-28. 
57. Xia, E.-Q.; Deng, G.-F.; Guom Y.-J.; Li, H.-B. Biological activities of polyphenols from grapes. Int. J. Mol. Sci. 2010, 11, 622-646.

58. Doshi, P.; Pandurang, A.; Banerjee, K. Phenolic composition and antioxidant activity in grapevine parts and berries (Vitis vinifera L.) cv. Kishmish Chornyi (Sharad Seedless) during maturation. Int. J. Food Sci. Technol. 2006, 41, 1-9.

59. Fernandes, F.; Ramalhosa, E.; Pires, P.; Verdial, J.; Valentão, P.; Andrade, P.; Bento, A.; Pereira, J.A. Vitis vinifera leaves towards bioactivity. Ind. Crops Prod. 2013, 43, 434-440.

60. Gurbuz, Y. Determination of nutritive value of leaves of several Vitis vinifera varieties as a source of alternative feedstuff for sheep using in vitro and in situ measurements. Small Rumin. Res. 2007, 71, 59-66.

61. Pérez-Serradilla, J.A.; Luque de Castro, M.D. Microwave-assisted extraction of phenolic compounds from wine lees and spray-drying of the extract. Food Chem. 2011, 124, 1652-1659.

62. Mazauric, J.P., Salmon, J.M. Interactions between yeast lees and wine polyphenols during simulation of wine aging. II. Analyses of desorbed polyphenol compounds from yeast lees. J. Agric. Food Chem. 2006, 54, 3876-3881.

63. Vattem, D.A.; Shetty, K. Ellagic acid production and phenolic antioxidant activity in cranberry pomace (Vaccinium macrocarpon) mediated by Lentinus edodes using a solid-state system. Process Biochem. 2003, 39, 367-379.

64. Tao, Y.; García, J.F.; Sun, D.W. Advances in wine aging technologies for enhancing wine quality and accelerating wine aging process. Crit. Rev. Food Sci. Nutr. 2014, 54, 817-835.

65. Anastasiadi, M.; Pratsinis, H.; Kletsas, D.; Skaltsounis, A.L.; Haroutounian, A. Grape stem extracts: Polyphenolic content and assessment of their in vitro antioxidant properties. Food Sci. Technol. 2012, 48, 316-322.

66. Naczk, M.; Shahidi, F. Extraction and analysis of phenolics in food. J. Chrom. A 2004, 1054, 95-111.

67. Kris-Etherton, P.M.; Hecker, K.D.; Bonanome, A.; Coval, S.M.; Binkoski, A.E.; Hilpert, K.F.; Griel, A.E.; Etherton, T.D. Bioactive compounds in food: Their role in the prevention of cardiovascular disease and cancer. Am. J. Med. 2002, 113, 71S-88S.

68. Garrido, J.; Borges, F. Wine and grape polyphenols-A chemical perspective. Food Res. Int. 2013, 54, 1844-1858.

69. Shahidi, F.; Naczk, M. Phenolics in Food and Nutraceuticals: Sources, Applications and Health Effects; CRC Press: Boca Raton, FL, USA, 2004.

70. Pridham, J.B. Phenolics in Plants in Health and Disease; Pergamon Press: New York, NY, USA, 1960.

71. Bengoechea M.L.; Sancho, A.I.; Bartolomé, B.; Estrella, I.; Gómez-Cordovés, C.; Hernández, T. Phenolic composition of industrially manufactured Purées and concentrates from peach and apple fruits. J. Agric. Food Chem. 1997, 45, 4071.

72. Gharras, H.E. Polyphenols: Food sources, properties and applications-A review. Int. J. Food Sci. Technol. 2009, 44, 2512-2518.

73. Kähkönen, M.P.; Hopia, A.I.; Vuorela, H.J.; Rauha, J.-P.; Pihlaja, K.; Kujala, T.S.; Heinonen, M. Antioxidant activity of plant extracts containing phenolic compounds. J. Agric. Food Chem. 1999, 47, 3954-3962. 
74. Berrin, B.; Kassel, G.; Derya, T. Study of polyphenol content in the seeds of red grape (Vitis vinifera L.) verities cultivated in Turkey and their antiradical activity. Food Chem. 2008, 56, 312-327.

75. Nilgün, G.B.; Gülcan, O.; Osman, S. Total phenolic contents and antibacterial activities of grape (Vitis vinifera L.) extracts. Food Control 2004, 15, 335-339.

76. De Freitas, V.A.P.; Glories, Y. Concentration and compositional changes of procyanidins in grape seeds and skin of white Vitis vinifera varieties. J. Sci. Food Agric. 1999, 79, 1601-1606.

77. Kennedy, J.A.; Matthews, M.A.; Waterhouse, A.L. Changes in grape seed polyphenols during ripening. Phytochemistry 2008, 55, 77-85.

78. Yilmaz, Y.; Toledo, R.T. Oxygen radical absorbance capacities of grape/wine industry byproducts and effect of solvent type on extraction of grape seed polyphenols. J. Food Comp. Anal. 2006, 19, $41-48$.

79. Zhang, Z.S.; Li, D.; Wang, L.J.; Ozkan, N.; Chen, X.D.; Mao, Z.H.; Yang, H.Z. Optimization of ethanol-water extraction of lignans from flaxseed. Sep. Purif. Technol. 2007, 57, 17-24.

80. Basha, S.M.; Musingo, M.; Colova Hasna, V.S. Compositional differences in the phenolics compounds of muscadine and bunch grape wines. Afr. J. Biotechnol. 2004, 3, 523-528.

81. Apostolou, A.; Stagos, D.; Galitsiou, E.; Spyrou, A.; Haroutounian, S.; Portesis, N.; Trizoglou, I.; Hayes, A.W.; Tsatsakis, A.M.; Kouretas, D. Assessment of polyphenolic content, antioxidant activity, protection against ROS-induced DNA damage and anticancer activity of Vitis vinifera stem extracts. Food Chem. Toxicol. 2013, 61, 60-68.

82. Di Lecce, G.; Arranz, S.; Jáuregui, O.; Tresserra-Rimbau, A.; Quifer-Rada, P.; Lamuela-Raventós, R.M. Phenolic profiling of the skin, pulp and seeds of Albariño grapes using hybrid quadrupole time-of-flight and triple-quadrupole mass spectrometry. Food Chem. 2014, $145,874-882$.

83. Kallithraka, S.; Salachaa, M.I.; Tzouroua, I. Changes in phenolic composition and antioxidant activity of white wine during bottle storage: Accelerated browning test versus bottle storage. Food Chem. 2009, 113, 500-505.

84. Montealegre, P.R.; Peces, R.R.; Vozmediano, J.L.C.; Gascueña, J.M.; Romero, G. Phenolic compounds in skins and seeds of ten grape Vitis vinifera varieties grown in a warm climate. J. Food Comp. Anal. 2006, 19, 687-693.

85. Singleton, V.L.; Timberlake, C.F.; Lea, A.G.H. The phenolic cinnamates of white grape. J. Sci. Food Agric. 1978, 29, 403.

86. Schoedl, K.; Schumacher, R.; Forneck, A. Studying the polyphenols of grapevine leaves according to age and insertion level under controlled conditions. Sci. Hortic. 2012, 141, 37-41.

87. Gómez-Alonso, S.; García-Romero, E.; Hermosín-Gutiérrez, I. HPLC analysis of diverse grape and wine phenolics using direct injection and multidetection by DAD and fluorescence. $J$. Food Compos. Anal. 2007, 20, 618-626.

88. Balasundram, N.; Sundram, K.; Samman, S. Phenolic compounds in plants and agroindustrial by-products: Antioxidant activity, occurrence, and potential uses. Food Chem. 2006, 99, 191-203.

89. Baderschneider, B.; Winterhalter, P. Isolation and characterization of novel benzoates, cinnamates, flavonoids, and lignans from Riesling wine and screening for antioxidant activity. J. Agric. Food Chem. 2001, 49, 2788-2798. 
90. Mattivi, F.; Guzzon, R.; Vrhovsek, U.; Stefanini, M.; Velasco, R. Metabolite profiling of grape: Flavonols and anthocyanins. J. Agric. Food Chem. 2006, 54, 7692-7702.

91. Cook, N.C.; Samman, S. Flavonoids-Chemistry, metabolism, cardioprotective effects, and dietary sources. J. Nutr. Biochem. 1996, 7, 66-76.

92. Çetin, E.S.; Altinöz, D.; Tarçan, E.; Baydar, N.G. Chemical composition of grape canes. Ind. Crops Prod. 2011, 34, 994-998.

93. Sá, M.; Justino, V.; Spranger, M.I.; Zhao, Y.Q.; Han, L.; Suna, B.S. Extraction yields and anti-oxidant activity of proanthocyanidins from different parts of grape pomace: Effect of mechanical treatments. Phytochem. Anal. 2014, 25, 134-140.

94. Mildner-Szkudlarz, S.; Bajerska, J.; Zawirska-Wojtasiaka, R.; Góreckac, D. White grape pomace as a source of dietary fibre and polyphenols and its effect on physical and nutraceutical characteristics of wheat biscuits. J. Sci. Food Agric. 2013, 93, 389-395.

95. Castillo-Muñoz, N.; Gómez-Alonso, S.; García-Romero, E.; Hermosín-Gutiérrez, I. Flavonol profiles of Vitis vinifera red grapes and their single-cultivar wines. J. Agric. Food Chem. 2007, 55, 992-1002.

96. Jeffery, D.W.; Parker, M.; Smith, P.A. Flavonol composition of Australian red and white wines determined by high-performance liquid chromatography. Aust. J. Grape Wine R. 2008, 14, $153-161$.

97. Ruberto, G.; Renda, A.; Daquino, C.; Amico, V.; Spatafora, C.; Tringali, C.; Tommasi, N. Polyphenol constituents and antioxidant activity of grape pomace extracts from five Sicilian red grape cultivars. Food Chem. 2007, 100, 203-210.

98. Amico, V.; Napoli, E.M.; Renda, A.; Ruberto, G.; Spatafora, C.; Tringali, C. Constituents of grape pomace from the Sicilian cultivar "Nerello Mascalese". Food Chem. 2004, 88, 599-607.

99. Amico, V.; Chillemi, R.; Mangiafico, S.; Spatafora, C.; Tringali, C. Polyphenol-enriched fractions from Sicilian grape pomace: HPLC-DAD analysis and antioxidant activity. Bioresour. Technol. 2008, 99, 5960-5966.

100. Bonilla, F.; Mayen, M.; Merida, J.; Medina, M. Extraction of phenolic compounds from red grape marc for use as food lipid antioxidants. Food Chem. 1999, 66, 209-215.

101. Castillo-Muñoz, N.; Gómez-Alonso, S.; García-Romero, E.; Gómez, M.V.; Velders, A.H.; Hermosín-Gutiérrez, I. Flavonol 3-O-glycosides series of Vitis vinifera cv. Petit Verdot red wine grapes. J. Agric. Food Chem. 2009, 57, 209-219.

102. Spatafora, C.; Barbagallo, E.; Amico, V.; Tringali, C. Grape stems from Sicilian Vitis vinifera cultivars as a source of polyphenol-enriched fractions with enhanced antioxidant activity. Food Sci. Technol. 2013, 54, 542-548.

103. González-Centeno, M.R.; Jourdes, M.; Fermenia, A.; Simal, S.; Rosselló, C.; Teissedre, P.-L. Proanthocyanidin composition and antioxidant potential of the stem winemaking byproducts from 10 different grape varieties (Vitis vinifera L.). J. Agric. Food Chem. 2012, 60, 11850-11858.

104. Ky, I.; Lorrain, B.; Kolbas, N.; Crozier, A.; Teissedre, P.L. Wine by-products: Phenolic characterization and antioxidant activity evaluation of grapes and grape pomaces from six different french grape varieties. Molecules 2014, 19, 482-506. 
105. Lago-Vanzela, E.S.; Pierotti Procópio, D.; Filomeno Fontes, E.A.; Mota Ramos, A.; Stringheta, P.C.; da-Silva, R.; Castillo-Muñoz, N.; Hermosín-Gutiérrez, I. Aging of red wines made from hybrid grape cv. BRS Violeta: Effects of accelerated aging conditions on phenolic composition, color and antioxidant activity. Food Res. Int. 2014, 56, 182-189.

106. Gómez-Plaza, E.; Gil-Munoz, R.; Lopez-Roca, J.M.; Martínez-Cutillas, A.; Fernández-Fernández, J.I. Phenolic compounds and color stability of red wines: Effect of skin maceration time. Am. J. Enol. Vitic. 2001, 52, 266-270.

107. Quiñones, M.; Guerrero, L.; Suarez, M.; Pons, Z.; Aleixandre, A.; Arola, L.; Muguerza, B. Low-molecular procyanidin rich grape seed extract exerts antihypertensive effect in males spontaneously hypertensive rats. Food Res. Int. 2013, 51, 587-595.

108. Jara-Palacios, M.J.; González-Manzano, S.; Escudero-Gilete, M.L.; Hernanz, D.; Dueñas, M.; González-Paramás, A.M.; Heredia, F.J.; Santos-Buelga, C. Study of zalema grape pomace: Phenolic composition and biological effects in Caenorhabditis elegans. J. Agric. Food Chem. 2013, 61, 5114-5121.

109. Ratnasooriya, C.C.; Rupasinghe, H.P.V. Extraction of phenolic compounds from grapes and their pomace using $\beta$-cyclodextrin. Food Chem. 2012, 134, 625-631.

110. Guendez, R.; Kallithraka, S.; Makris, D.P.; Kefalas, P. Determination of low molecular weight phenolic constituents in grape (Vitis vinifera sp.) seed extracts: Correlation with antiradical activity. Food Chem. 2005, 89, 1-9.

111. Jeandet, P.; Bessis, R.; Gautheron, B. The production of resveratrol (3,5,4'-trihydroxystilbene) by grape berries in different developmental stages. Am. J. Enol. Vitic. 1991, 42, 41-46.

112. Treutter, D. Significance of flavonoids in plant resistance: A review. Environ. Chem. Lett. 2006, 4, 147-157.

113. Burdock, G.A. Fenaroli's Handbook of Flavor Ingredients, 5th ed.; CRC Press: Boca Raton, FL, USA, 2005.

114. Weber, H.A.; Hodges, A.E.; Guthrie, J.R.; O’Brien, B.M.; Robaugh, D.; Clark, A.P.; Harris, R.K.; Algaier, J.W.; Smith, C.S. Comparison of proanthocyanidins in commercial antioxidants: Grape seed and pine bark extracts. J. Agric. Food Chem. 2007, 55, 148-156.

115. Monagas, M.; Mez-Cordoveä, C.G.; Bartolomeä, B.; Laureano, O.; Ricardo da Silva, J.M. Monomeric, oligomeric, and polymeric flavan-3-ol composition of wines and grapes from Vitis Vinifera L. cv. Graciano, Tempranillo, and Cabernet Sauvignon. J. Agric. Food Chem. 2003, 51, 6475-6781.

116. De Freitas, V.A.P.; Glories, Y.; Monique, A. Development changes of procyanidines in grapes of red Vitis vinifera varieties and their composition in respective wines. Am. J. Enol. Vitic. 2000, 51, 397-403.

117. De Pascual-Teresa, S.; Moreno, D.A.; García-Viguera, C. Flavanols and anthocyanins in cardiovascular health: A review of current evidence. Int. J. Mol. Sci. 2010, 11, 1679-1703.

118. Castañeda-Ovando, A.; Pacheco-Hernández, M.L.; Páez-Hernández, M.E.; Rodríguez, J.A.; Galán-Vidal, C.A. Chemical studies of anthocyanins: A review. Food Chem. 2009, 13, 859-871. 
119. Teixeira Barcia, M.; Becker Pertuzatti, P.; Rodrigues, D.; Gómez-Alonso, S.; Hermosín-Gutiérrez, I.; Teixeira Godoy, H. Occurrence of low molecular weight phenolics in Vitis vinifera red grape cultivars and their winemaking by-products from São Paulo (Brazil). Food Res. Int. 2014, 62, 500-513.

120. Pérez-Serradilla, J.A.; Castro de Luque, M.D. Role of lees in wine production: A review. Food Chem. 2008, 111, 447-456.

121. Liu, C.; Wang, L.; Wang, J.; Wu, B.; Liu, W.M.; Fan, P.; Liang, Z.; Li, S. Resveratrols in Vitis berry skins and leaves: Their extraction and analysis by HPLC. Food Chem. 2013, 136, 643-649.

122. Lima, M.T.R.; Waffo-Téguo, P.; Teissedre, P.L.; Pujolas, A.; Vercauteren, J.; Cabanis, J.C.; Mérillon, J.M. Determination of stilbenes (trans-astringin, cis- and trans-piceid, and cis- and trans-resveratrol) in Portuguese wines. J. Agric. Food Chem. 1999, 47, 2666-2670.

123. Moreno-Labanda, J.F.; Mallavia, R.; Pérez-Fons, L.; Lizama, V.; Saura, D.; Micol, V. Determination of piceid and resveratrol in Spanish wines deriving from Monastrell (Vitis vinifera L.) grape variety. J. Agric. Food Chem. 2004, 52, 5396-5403.

124. Püssa, T.; Floren, J.; Kuldkepp, P.; Raal, A. Survey of grapevine Vitis vinifera stem polyphenols by liquid chromatography-diode array detection-tandem mass spectrometry. J. Agric. Food Chem. 2006, 54, 7488-7494.

125. Gonzalez-Barrio, R.; Beltran, D.; Cantos, E.; Gil, M.; Espin, J.; Tomas-Barberan, F. Comparison of ozone and UV-C treatments on the postharvest stilbenoid monomer, dimer, and trimer induction in var. "Superior" white table grapes. J. Agric. Food Chem. 2006, 54, 4222-4228.

126. Li, X.; Wu, B.; Wang, L.; Li, S. Extractable amounts of trans-resveratrol in seed and berry skin in Vitis evaluated at the germplasm level. J. Agric. Food Chem. 2006, 54, 8804-8811.

127. Kalantari, H.; Das, D.K. Physiological effects of resveratrol. Biofactors 2010, 36, 401-406.

128. Yilmaz, E.E.; Ozvural, E.B.; Vural, H. Extraction and identification of proanthocyanidins from grape seed (Vitis vinifera) using supercritical carbon dioxide. J. Supercrit. Fluids 2011, 55, 924-928.

129. Cheynier, V. Polyphenols in foods are more complex than often thought. Am. J. Clin. Nutr. 2005, 81, 223S-229S.

130. Proestos, C.; Bakogiannis, A.; Psarianos, C.; Koutinas, A.A.; Kanellaki, M.; Komaitis, M. High performance liquid chromatography analyses of phenolic substances in Greek. Food Control. 2005, 16, 319-323.

131. Ames, B.N. Endogenous oxidative DNA damage, aging and cancer. Free Rad. Res. Commun. 1989, 7, 121-122.

132. Willet, W.C. Diet and health: What should we eat? Science 1994, 264, 532-537.

133. Hertog, M.G.L.; Hollman, P.C.H.; Katan, M.B. Content of potentially anticarcinogenic flavonoids of 28 vegetables and 9 fruits commonly consumed in the Netherlands. J. Agric. Food Chem. 1992, 40, 2379-2383.

134. Rimm, E.B.; Stampfer, M.J.; Asherio, A.; Giovannucci, E.; Colditz, G.A.; Willett, W.C. Vitamin E consumption and the risk of coronary heart disease in men. N. Engl. J. Med. 1993, $328,1450-1456$.

135. Esterbauer, H.; Geibicki, J.; Puhl, H.; Jürgens, G. The role of lipid peroxidation and antioxidants in oxidative modification of LDL. Free Radic. Biol. Med. 1992, 13, 341-390. 
136. Jalial, I.; Grundy, S.M. Effects of dietary supplementation with a-tocopherol on the oxidative modification of low-density lipoprotein. J. Lipid Res. 1992, 33, 899-906.

137. Kinsella, J.E.; Frankel, E.; German, B.; Kanner, J. Possible mechanism for the protective role of the antioxidant in wine and plant foods. Food Technol. 1993, 47, 85-89.

138. Jakobek, L.; Sěruga, M.; Sěruga, B.; Novak, I.; Medvidović-Kosanovic, M. Phenolic compound composition and antioxidant activity of fruits of Rubus and Prunus species from Croatia. Int. J. Food Sci. Technol. 2009, 44, 860-868.

139. Jacob, J.K.; Hakimuddin, F.; Paliyath, G.; Fisher, H. Antioxidant and antiproliferative activity of polyphenols in novel high-polyphenol grape lines. Food Res. Int. 2008, 41, 419-428.

140. Soobrattee, M.A.; Neergheena, V.S.; Luximon-Rammaa, A.; Aruomab, O.I.; Bahoruna, T. Phenolics as potential antioxidant therapeutic agents: Mechanism and actions. Mutat. Res. Fundam. Mol. Mech. Mutagen. 2005, 579, 200-213.

141. Di Majo, D.; Guardia, M.; Giammanco, S.; Neve, L.; Giammanco, M. The antioxidant capacity of red wine in relationship with its polyphenolic constituents. Food Chem. 2008, 111, 45-49.

142. Spranger, I.; Sun, B.S.; Mateus, A.M.; de Freitas, V.; Ricardo-da-Silva, A.M. Chemical characterization and antioxidant activities of oligomeric and polymeric procyanidin fractions from grape seeds. Food Chem. 2008, 108, 519-532.

143. Dajanta, K.; Janpum, P.; Leksing, W. Antioxidant capacities, total phenolics and flavonoids in black and yellow soybeans fermented by Bacillus subtilis: A comparative study of Thai fermented soybeans (thua nao). Int. Food Res. J. 2013, 20, 3125-3132.

144. Bobek, P. Dietary tomato and grape pomace in rats: Effect on lipids in serum and liver, and on antioxidant status. Br. J. Biomed. Sci. 1999, 56, 109-113.

145. Dohadwala, M.M.; Vita, J.A. Grapes and cardiovascular disease. J. Nutr. 2009, 139, 1788S-1793S.

146. Shi, J.; Yu, J.; Pohorly, J.E.; Young, C.J.; Bryan, M.; Wu, Y. Optimization of the extraction of polyphenols from grape seed meal by aqueous ethanol solution. J. Food Agric. Environ. 2003, 1, 42-47.

147. Moreno, D.A.; Ilic, N.; Poulev, A.; Brasaemle, D.L.; Fried, S.K.; Raskin, I. Inhibitory effects of grape seed extract on lipases. Nutrition 2003, 19, 876-879.

148. Sano, T.; Oda, E.; Yamashita, T.; Naemura, A.; Yamakoshi, J.; Yamamoto, J. Anti-thrombotic effect of proanthocyanidin, a purified ingredient of grape seed. Thromb. Res. 2005, 115, 115-121.

149. Bagchi, D.; Bagchi, M.; Stohs, S.J.; Das, D.K.; Ray, S.D.; Kuscinski, C.A.; Joshi, S.S.; Pruess, H.G. Free radicals and grape seed proanthocyanidin extract, importance in human health and disease prevention. Toxicology 2000, 148, 187-197.

150. Karthikeyan, K.; Sarala Bai, B.R.; Devaraj, S.N. Cardioprotective effect of grape seed procyanidins on isoproernol-induced myocardial injury in rats. J. Cardiovasc. Pharm. 2009, 53, $109-115$.

151. Olas, B.; Wachowicz, B.; Tomczak, A.; Erler, J.; Stochmal, A.; Oleszek, W. Comparative antiplatelet and antioxidant properties of polyphenol-rich extracts from: Berries of Aronia melanocarpa, seeds of grape and bark of Yucca schidigera in vitro. Platelets 2008, 19, 70-77.

152. Álvarez, E.; Rodiño-Janeiro, B.K.; Jerez, M.; Ucieda-Somoza, R.; Núñez, M.J.; González-Juanatey, J.R. Procyanidins from grape pomace are suitable inhibitors of human endothelial NADPH oxidase. J. Cell Biochem. 2012, 113, 1386-1396. 
153. Jayaprakasha, G.K.; Selvi, T.; Sakaria, K.K. Antibacterial and antioxidant activities of grape (Vitis vinifera) seed extracts. Food Res. Int. 2003, 36, 17-122.

154. Mendoza, L.; Yañez, K.; Vivanco, M.; Melo, R.; Cotoras, M. Characterization of extracts from winery by-products with antifungal activity against Botrytis cinerea. Ind. Crops Prod. 2013, 43, 360-364.

155. Anastasiadi, M.; Chorianopoulos, N.G.; Nychas, G.J.E.; Haroutounian, S.A. Antilisterial activities of polyphenol-rich extracts of grapes and vinification byproducts. J. Agric. Food Chem. 2009, 57, 457-463.

156. Vaquero, M.J.R.; Alberto, M.R.; Manca de Nadra, M.C. Antibacterial effect of phenolic compounds from different wines. Food Control. 2007, 18, 93-101.

157. Rhodes, P.L.; Mitchell, J.W.; Wilson, M.W.; Melton, L.D. Antilisterial activity of grape juice and grape extracts derived from Vitis vinifera variety Ribier. Int. J. Microb. 2006, 107, 281-286.

158. Ahn, J.; Grün, I.U.; Mustapha, A. Antimicrobial and antioxidant activities of natural extracts in vitro and in ground beef. J. Food Prot. 2004, 67, 148-155.

159. Ahn, J.; Grün, I.U.; Mustapha, A. Effects of plant extracts on microbial growth, color change, and lipid oxidation in cooked beef. Food Microbiol. 2007, 24, 7-14.

160. Jayaprakasha, G.K.; Signh, R.P.; Sakariah, K.K. Antioxidant activity of grape seed (Vitis vinifera) extracts on peroxidation models in vitro. Food Chem. 2001, 73, 285-290.

161. Paulo, L.; Ferreira, S.; Gallardo, E.; Queiroz, J.A.; Domingues, F. Antimicrobial activity and effects of resveratrol on human pathogenic bacteria. World J. Microbiol. Biotechnol. 2010, 26, $1533-1538$.

162. Mitjans, M.; del Campo, J.; Abajo, C.; Martínez, V.; Selga, A.; Lozano, C.; Torres, J.L.; Vinardell, M.P. Immunomodulatory activity of a new family of antioxidants obtained from Grape polyphenols. J. Agric. Food Chem. 2004, 52, 7297-7299.

163. Li, X.L.; Cai, Y.Q.; Qin, H.; Wu, Y.J. Therapeutic effect and mechanism of proanthocyanidins from grape seeds in rats with TNBS-induced ulcerative colitis. Can. J. Physiol. Pharmacol. 2008, 86, 841-849.

164. Cheah, K.Y.; Bastian, S.E.; Acott, T.M.; Abimosleh, S.M.; Lymn, K.A.; Howarth, G.S. Grape seed extract reduces the severity of selected disease markers in the proximal colon of dextran sulphate sodium-induced colitis in rats. Dig. Dis. Sci. 2013, 58, 970-977.

165. Wang, Y.H.; Ge, B.; Yang, X.L.; Zhai, J.; Yang, L.N.; Wang, X.X.; Liu, X.; Shi, J.C.; Wu, Y.J. Proanthocyanidins from grape seeds modulates the nuclear factor- $\kappa \mathrm{B}$ signal transduction pathways in rats with TNBS-induced recurrent ulcerative colitis. Int. Immunopharmacol. 2011, $11,1620-1627$.

166. Wang, Y.H.; Yang, X.L.; Wang, L.; Cui, M.X.; Cai, Y.Q.; Li, X.L.; Wu, Y.J. Effects of proanthocyanidins from grape seed on treatment of recurrent ulcerative colitis in rats. Can. J. Physiol. Pharmacol. 2010, 88, 888-898.

167. Alexander, R.W. Hypertension and the pathogenesis of atherosclerosis: Oxidative stress and the mediation of arterial inflammatory response: A new perspective. Hypertension 1995, 25, 155-161.

168. Kritchevsky, S.B.; Bush, A.J.; Pahor, M.; Gross, M.D. Serum carotenoids and markers of inflammation in nonsmokers. Am. J. Epidemiol. 2000, 152, 1065-1071. 
169. Brighenti, F.; Valtuena, S.; Pellegrini, N.; Ardigo, D.; del Rio, D.; Salvatore, S.; Piatti, P.; Serafini, M.; Zavaroni, I. Total antioxidant capacity of the diet is inversely and independently related to plasma concentration of high-sensitivity C-reactive protein in adult Italian subjects. Br. J. Nutr. 2005, 93, 619-625.

170. Hogan, S.; Canning, C.; Sun, S.; Sun, X.; Zhou, K. Effects of grape pomace antioxidant extract on oxidative stress and inflammation in diet induced obese mice. J. Agric. Food Chem. 2010, 58, $11250-11256$.

171. Feng, Y.; Liu, Y.M.; Fratkins, J.; Leblanc, M. Grape seed extract suppresses lipid peroxidation and reduces hypoxic ischemic brain injury in neonatal rats. Brain Res. Bull. 2005, 66, 120-127.

172. Balu, M.; Sangeetha, P.; Murali, G.; Panneerselvam, C. Modulatory role of grape seed extract on age-related oxidative DNA damage in central nervous system of rats. Brain Res. Bull. 2006, 68, 469-473.

173. Markus, M.A.; Morris, B.J. Resveratrol in prevention and treatment of common clinical conditions of aging. Clin. Interv. Aging 2008, 3, 331-339.

174. Fontana, A.R.; Antoniolli, A.; Bottini, R. Grape pomace as a sustainable source of bioactive compounds: Extraction, characterization, and biotechnological applications of phenolics. J. Agric. Food Chem. 2013, 61, 8987-9003.

175. Agustin-Salazar, S.; Medina-Juárez, L.A.; Soto-Valdez, H.; Manzanares-López, F.; Gámez-Meza, N. Influence of the solvent system on the composition of phenolic substances and antioxidant capacity of extracts of grape (Vitis vinifera L.) marc. Aust. J. Grape Wine R. 2014, doi:10.1111/ajgw.12063.

176. Spigno, G.; de Faveri, D.M. Antioxidants from grape stalks and marc: Influence of extraction procedure on yield, purity and antioxidant power of the extracts. J. Food Eng. 2007, 78, 793-801.

177. Lapornik, B.; Prošek, M.; Golc Wondra, A. Comparison of extracts prepared from plant by-products using different solvents and extraction time. J. Food Eng. 2005, 71, 214-222.

178. EFSA. Scientific opinion on the evaluation of the substances currently on the list in the Annex to Commission Directive 96/3/EC as acceptable previous cargoes for edible fats and oils_-Part I of III. EFSA J. 2011, 9, 2482.

179. Joint FAO/WHO Expert Committee on Food Additives. Toxicological evaluation of some extraction solvents and certain other substances. In Proceedings of the FAO Nutrition Meetings; WHO: Geneva, Switzerland, 1970.

180. Cruz, J.M.; Domínguez, H.; Parajó, J.C. Assessment of the production of antioxidants from winemaking waste solids. J. Agric. Food Chem. 2004, 52, 5612-5620.

181. Bucić-Kojić, A.; Planinić, M.; Tomas, S.; Bilić, M.; Velić, D. Study of solid-liquid extraction kinetics of total polyphenols from grape seeds. J. Food Eng. 2007, 81, 236-242.

182. Marqués, J.L.; della Porta, G.; Reverchon, E., Renuncio, J.A.R.; Mainar, A.M. Supercritical antisolvent extraction of antioxidants from grape seeds after vinification. J. Supercrit. Fluid 2013, 82, 238-243.

183. Fang, Z.; Bhandari, B. Encapsulation of polyphenols-A review. Trends Food Sci Technol. 2010, 21, 510-523. 
184. Alonso, E.; Revilla, E.; Kovac, V.; Pekic, B. Study of the extraction of proanthocyanidins from grape seeds. Food Chem. 1998, 61, 201-206.

185. Spigno, G.; Tramelli, L.; de Faveri, D.M. Effects of extraction time, temperature and solvent on concentration and antioxidant activity of grape marc phenolics. J. Food Eng. 2007, 81, 200-208.

186. Dominguez-Perles, R.; Teixeira, A.; Rosa, E.; Barros, A. Assessment of (poly)phenols in grape (Vitis vinifera L.) stems by using food/pharma industry compatible solvents and response surface methodology. Food Chem. 2014, 164, 339-346.

187. Novak, I.; Janeiro, P.; Seruga, M.; Oliveira-Brett, A.M. Ultrasound extracted flavonoids from four varieties of Portuguese red grape skins determined by reverse-phase high-performance liquid chromatography with electrochemical detection. Anal. Chim. Acta 2008, 630, 107-115.

188. Liazid, A.; Guerrero, R.F.; Cantos, E.; Palma, M.; Barroso, C.G. Microwave assisted extraction of anthocyanins from grape skins. Food Chem. 2011, 124, 1238-1243.

189. Concota, A.; Cincota, A.H. For threapy of atherosclerotic cardiovascular disease. WO 20010510 88A1; U.S. Patent US 20020146400 A1, 10 October 2002.

190. Bekhit, A.E.D.A.; Cheng, V.J.; McConnell, M.; Zhao, J.H.; Sedcole, R.; Harrison, R. Antioxidant activities, sensory and anti-influenza activity of grape skin tea infusion. Food Chem. 2011, 129, 837-845.

191. Perumalla, A.V.S.; Hettiarachchy, N.S. Green tea and grape seed extracts-Potential applications in food safety and quality. Food Res. Int. 2011, 44, 827-839.

192. Characterization and Functional and Safety Evaluation of Bioactive Compounds from Iberian-American Fruits for Food Ingredients (CORNUCOPIA). Thematic Network CYTED Ref. 112RT0460. Available online: http://redcornucopia.org (accessed on 2 September 2014).

(C) 2014 by the authors; licensee MDPI, Basel, Switzerland. This article is an open access article distributed under the terms and conditions of the Creative Commons Attribution license (http://creativecommons.org/licenses/by/3.0/). 